\title{
Support and Rank Varieties of Totally Acyclic Complexes
}

\author{
by \\ NATHAN THOMAS STEELE
}

Presented to the Faculty of the Graduate School of

The University of Texas at Arlington in Partial Fulfillment

of the Requirements

for the Degree of

DOCTOR OF PHILOSOPHY

THE UNIVERSITY OF TEXAS AT ARLINGTON

August 2016 
Copyright @ by Nathan Thomas Steele 2016

All Rights Reserved 


\section{Acknowledgments}

I would like to thank my advisor, Dr. David Jorgensen, for his help and encouragement in preparing this dissertation and the algebra seminar group at UTA for the many conversations that helped point me in the right direction. I also thank my friends and family, without whom I could never have completed my studies.

July 15, 2016 


\section{Abstract \\ Support and Rank Varieties of Totally Acyclic Complexes}

Nathan Thomas Steele, Ph.D.

The University of Texas at Arlington, 2016

Supervising Professor: David Jorgensen

Support and rank varieties of modules over a group algebra of an elementary abelian $p$-group have been well studied. In particular, Avrunin and Scott showed that in this setting, the rank and support varieties are equivalent. Avramov and Buchweitz proved an analogous result for pairs of modules over arbitrary commutative local complete intersection rings. In this dissertation we study support and rank varieties in the triangulated category of totally acyclic chain complexes over a complete intersection ring and show that these varieties are also equivalent. We also show that any homogeneous affine variety is realizable as the support of some pair of totally acyclic complexes. 


\section{Table of Contents}

Acknowledgments .......................... iii

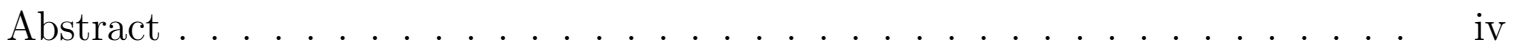

Chapter Page

Introduction . . . . . . . . . . . . . . . . . . . . . vii

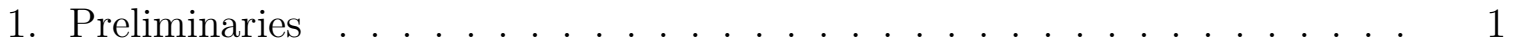

1.1 Triangulated Categories . . . . . . . . . . . . . . . 1

1.2 Chain Complexes . . . . . . . . . . . . . . . 6

1.3 The Triangulated Category $\mathrm{K}_{\mathrm{tac}}(R) \ldots \ldots \ldots$

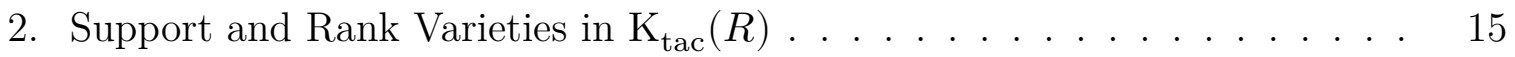

2.1 Eisenbud and Cohomology Operators in $\mathrm{K}_{\mathrm{tac}}(R) \ldots \ldots . \ldots$

2.2 Definitions of Support and Rank Varieties . . . . . . . . . . . . 20

3. Equivalence of Support and Rank Varieties . . . . . . . . . . . . . . . . . . 24

3.1 Finite Generation . . . . . . . . . . . . . . . . . . 24

3.2 Avrunin-Scott for $\mathrm{K}_{\mathrm{tac}}(R) \ldots \ldots \ldots$. . . . . . . . . . . . 32

3.3 Properties of Support/Rank Varieties . . . . . . . . . . . . . . . . 33

3.4 Rank Varieties of Higher Dimensions . . . . . . . . . . . . . . . . 36

3.5 Examples . . . . . . . . . . . . . . . . . . . 38

4. Realizability . . . . . . . . . . . . . . . . . . 42

4.1 Constructing a Sequence of Mapping Cones . . . . . . . . . . . . 43

4.2 Nilpotent Eisenbud Operators . . . . . . . . . . . . . 51

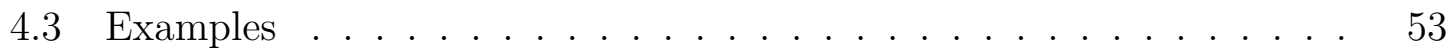

Conclusions and Future Work . . . . . . . . . . . . . . . . . . 61 


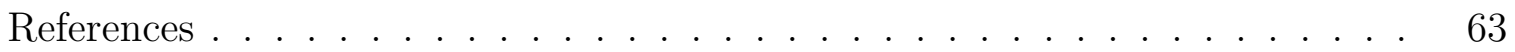

Biographical Statement . . . . . . . . . . . . . . 65 


\section{Introduction}

The main goal of this dissertation is to explore a theory of support and rank varieties in the triangulated category of totally acyclic complexes, $\mathrm{K}_{\mathrm{tac}}(R)$, over a complete intersection ring, $R=Q /\left(f_{1}, \ldots, f_{c}\right)$. We are interested in this category for

two main reasons. First, $\mathrm{K}_{\mathrm{tac}}(R)$ is a natural setting for the study of cohomology operators using Eisenbud's construction [15]. Many of the proofs involving support varieties in this category boil down to explicit computations involving the Eisenbud operators. In particular, we focus heavily on the mapping cones of these operators. Secondly, Buchweitz [12] showed that for any Gorenstein $\operatorname{ring} R, \mathrm{~K}_{\text {tac }}(R)$ is equivalent to several other triangulated categories. These include the singularity category and the stable category of maximal Cohen-Macaulay modules. Furthermore, if $R$ is a hypersurface ring, then $\mathrm{K}_{\mathrm{tac}}(R)$ is equivalent to the category of matrix factorizations. These categories have known applications in homological mirror symmetry and Landau-Ginzberg models.

In [19], Dan Quillen first introduced the idea of identifying algebraic varieties to cohomology rings of certain group algebras. Jon Carlson [13] then defined support and rank varieties for modules over group algebras of elementary abelian $p$-groups. Carlson was able to show that the rank variety was contained in the support variety and conjectured that these varieties are equal. Shortly thereafter, Avrunin and Scott $[6,1.1]$ were able to prove this conjecture. Avramov and Buchweitz [2, 2.5] later proved a similar result for pairs of modules over a complete intersection ring and they referred to both sets as the support variety. Benson, Iyengar, and Krause [7] then gave a generic construction for support varieties in triangulated categories using 
local cohomology and they applied this construction to the homotopy category of complexes of injective modules. More recently, Petter Bergh [8] showed that any homogeneous affine variety is realizable as the support of some pair of modules over a complete intersection ring. Avramov and Iyengar [4] showed this result also holds in the derived category.

In chapter one, we give background information about $\mathrm{K}_{\mathrm{tac}}(R)$. We state the definitions of a triangulated category and subcategory and discuss the triangulated structure of the homotopy category of chain complexes, $\mathrm{K}(R)$. We then show that the category of totally acyclic complexes is a triangulated subcategory of $\mathrm{K}(R)$.

In chapter two, we present an explicit and tractable construction of cohomology operators and of support and rank varieties in $\mathrm{K}_{\mathrm{tac}}(R)$. We take advantage of the triangulated structure of this category in chapter three to give what we feel are elegant and natural proofs of the classic properties of these varieties. In particular, we describe an iterated lifted mapping cone and use it to show that the rank and support varieties of a pair of totally acyclic complexes are equal, translating the Avrunin-Scott theorem to $\mathrm{K}_{\mathrm{tac}}(R)$.

Lastly, we discuss the realizability of support varieties in $\mathrm{K}_{\mathrm{tac}}(R)$ in chapter four. Starting with a complete resolution of the residue field $\mathbb{k}$, we construct a sequence of mapping cones of cohomology operators to create a totally acyclic complex whose support is a desired affine cone. We also discuss the existence of nilpotent cohomology operators and compute a few simple examples of support varieties. 


\section{Chapter 1}

\section{Preliminaries}

In this chapter we provide the basic definitions of triangulated categories and chain complexes. We also discus exactness and tensor products of chain complexes. We then describe the homotopy category of chain complexes, $\mathrm{K}(R)$ as well as the subcategory of totally acyclic complexes, $\mathrm{K}_{\mathrm{tac}}(R)$.

\subsection{Triangulated Categories}

Verdier [21] originally provided the definitions and basic results for triangulated categories in his thesis. A triangulated category is a category along with an endofunctor $\Sigma$ (often referred to as the shift or translation functor) and a class of diagrams called distinguished triangles. These triangles serve a similar role to that of short exact sequences in abelian categories. Triangulated categories serve as a natural setting for the study of cohomology.

Definition 1.1.1. (Rotman [20, 5.5]) A category $\mathcal{C}$ is called additive if

- $\operatorname{Hom}(A, B)$ is an abelian group for every $A, B \in \mathcal{C}$,

- given morphisms

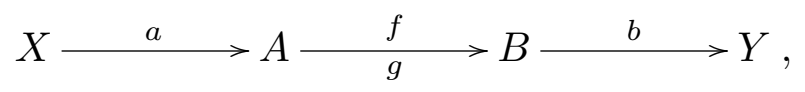

where $X, Y \in \mathcal{C}$, then

$$
b(f+g)=b f+b g \text { and }(f+g) a=f a+g a,
$$

- $\mathcal{C}$ has a zero object,

- $\mathcal{C}$ has finite products and finite coproducts. 
An additive category is furthermore called abelian if

- every morphism has a kernel and a cokernel,

- every monomorphism is a kernel and every epimorphism is a cokernel.

Definition 1.1.2. (Neeman $[18,1.1 .1])$ Let $\mathcal{C}$ be an additive category, $\Sigma: \mathcal{C} \rightarrow \mathcal{C}$ be an invertible functor, and $X, Y, Z$ be objects in $\mathcal{C}$. A candidate triangle in $\mathcal{C}$ with respect to $\Sigma$ is a diagram of the form

$$
X \stackrel{u}{\longrightarrow} Y \stackrel{v}{\longrightarrow} Z \stackrel{w}{\longrightarrow} \Sigma X
$$

such that $v \circ u, w \circ v$, and $\Sigma u \circ w$ are all the zero morphism.

Definition 1.1.3. (Neeman $[18,1.1 .2,1.4 .6])$ Let $\mathcal{T}$ be an additive category with an invertible endofunctor $\Sigma$ and a class of candidate triangles called distinguished triangles. We call $\mathcal{T}$ a triangulated category if the distinguished triangles satisfy the following axioms:

- TR0 Any candidate triangle which is isomorphic to a distinguished triangle is distinguished. The candidate triangle

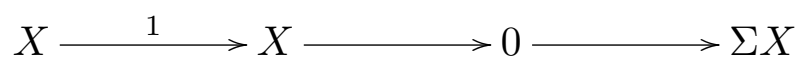

is distinguished.

- TR1 For any morphism $u: X \rightarrow Y$ in $\mathcal{T}$, there exists a distinguished triangle of the form

$$
X \longrightarrow \text { u } Y \longrightarrow Z \longrightarrow \Sigma X
$$

- TR2 The candidate triangle

$$
X \stackrel{u}{\longrightarrow} Y \stackrel{v}{\longrightarrow} Z \stackrel{w}{\longrightarrow} \Sigma X
$$

is distinguished if and only if

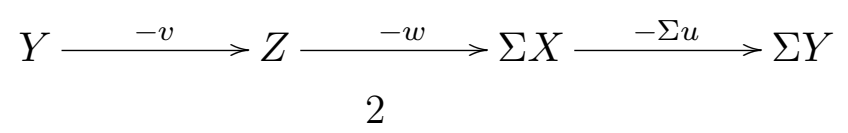


is distinguished.

- TR3 For any commutative diagram

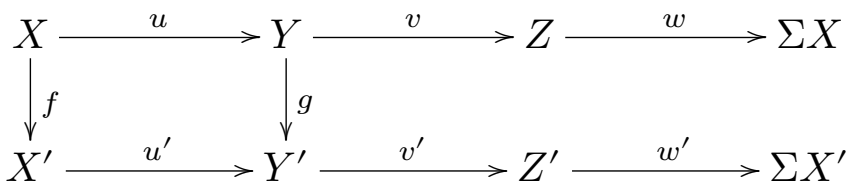

where the rows are distinguished triangles, there exists a morphism $h: Z \rightarrow Z^{\prime}$ such that the diagram

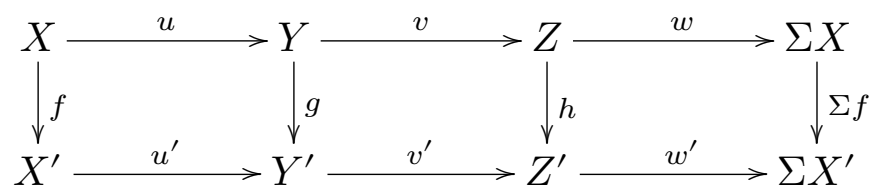

is commutative.

- TR4 Let $u: X \rightarrow Y$ and $v: Y \rightarrow Y^{\prime}$ be composable morphisms in $\mathcal{T}$. If the candidate triangles

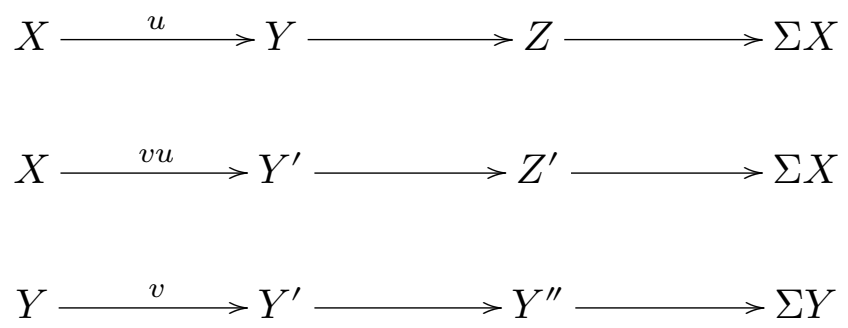

are distinguished, then we have a commutative diagram

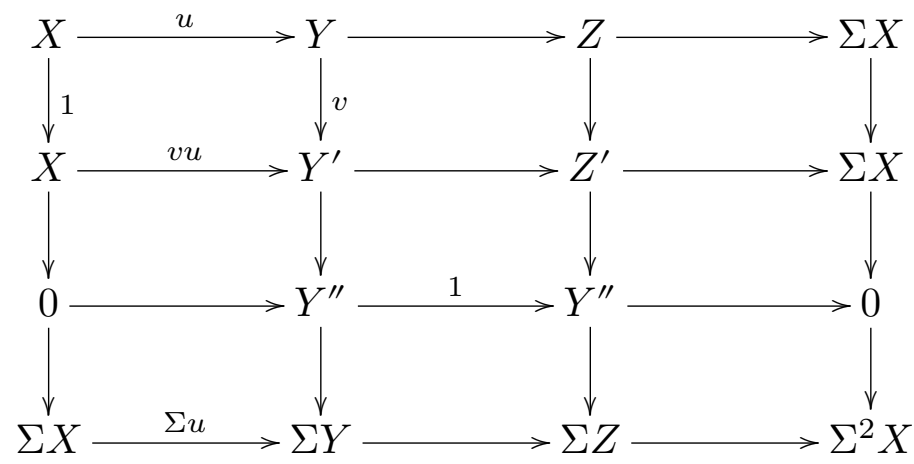

where every column and every row is a distinguished triangle. 
The fourth axiom is known as the octahedral axiom as the diagram can be represented as an octahedron where all of the faces commute.

Definition 1.1.4. Let $\mathcal{T}$ be a triangulated category. A full additive subcategory $\mathcal{S}$ in $\mathcal{T}$ is called a triangulated subcategory if every object isomorphic to an object in $\mathcal{S}$ is in $\mathcal{S}, \Sigma \mathcal{S}=\mathcal{S}$, and if for any distinguished triangle

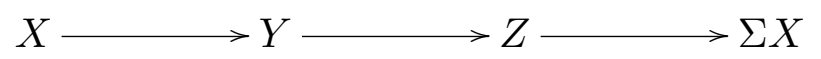

such that the objects $X$ and $Y$ are in $\mathcal{S}$, the object $\mathcal{Z}$ is also in $\mathcal{S}$.

A classic result of homological algebra states that any short exact sequence of complexes yields long exact sequences in homology [22, 1.3.1]. The following result translates this to the setting of triangulated categories.

Proposition 1.1.5. (Neeman [18, 1.1.10, 1.1.11]) Let $\mathcal{T}$ be a triangulated category and

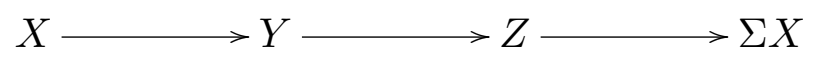

be a distinguished triangle in $\mathcal{T}$. Then applying the functors $\operatorname{Hom}(U, \ldots)$ and $\operatorname{Hom}(\ldots, U)$ to the above triangle yield long exact sequences

$\cdots \longrightarrow \operatorname{Hom}(U, X) \longrightarrow \operatorname{Hom}(U, Y) \longrightarrow \operatorname{Hom}(U, Z) \longrightarrow \operatorname{Hom}(U, \Sigma X) \longrightarrow \cdots$

and

$\cdots \lessdot \operatorname{Hom}(X, U) \longleftarrow \operatorname{Hom}(Y, U) \longleftarrow \operatorname{Hom}(Z, U) \longleftarrow \operatorname{Hom}(\Sigma X, U) \longleftarrow \cdots$

respectively.

The following proposition describes a family of triangulated categories formed from semi-simple abelian categories, i.e. abelian categories in which every short exact sequence splits. A simple example is the category of vector spaces over a field $\mathbb{k}$. We provide a sketch of Fritz's proof for illustrative purposes. 
Proposition 1.1.6. (Fritz $[16,8.1])$ Let $\mathcal{A}$ be an abelian category such that for any short exact sequence of objects

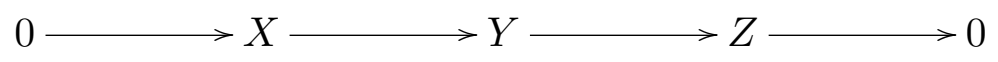

in $\mathcal{A}$, we have $Y \cong X \oplus Z$, i.e. all short exact sequences in $\mathcal{A}$ split. Also let $\Sigma$ be the identity endofunctor $\Sigma X=X$ for any $X \in \mathcal{A}$. For any candidate triangle

$$
X \stackrel{u}{\longrightarrow} Y \stackrel{v}{\longrightarrow} Z \stackrel{w}{\longrightarrow} X
$$

call the triangle distinguished if the sequence is exact at $X, Y, Z$ where exactness at $X$ means $\operatorname{Ker}(u)=\operatorname{Coker}(w)$. Then $\mathcal{A}$ is a triangulated category .

Proof. - TR0 Any sequence isomorphic to an exact sequence is itself exact. Furthermore, the sequence

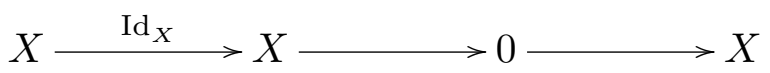

is exact since $\operatorname{Id}_{X}$ is an isomorphism.

- TR1 Let $u: X \rightarrow Y$ be a morphism in $\mathcal{A}, \pi_{u}$ be the natural projection onto $\operatorname{Coker}(u)$, and $\iota_{u}$ be the natural inclusion from $\operatorname{Ker}(u)$. Then the sequence

$$
X \stackrel{u}{\longrightarrow} Y \stackrel{\left(\begin{array}{c}
0 \\
\pi_{u}
\end{array}\right)}{\longrightarrow} \operatorname{Ker}(u) \oplus \operatorname{Coker}(u) \stackrel{\left(0 \iota_{u}\right)}{\longrightarrow} X
$$

is exact.

- TR2 Shifts of distinguished triangles are clearly distinguished since the shift functor is the identity.

- TR3 The diagram

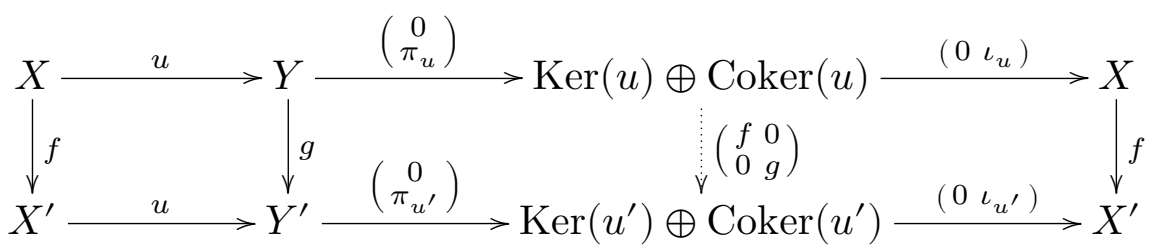

commutes. 
- TR4 Let $u: X \rightarrow Y$ and $v: Y \rightarrow Z$ be morphisms in $\mathcal{A}$. Consider the canonical morphisms from the universal properties of kernel and cokernel

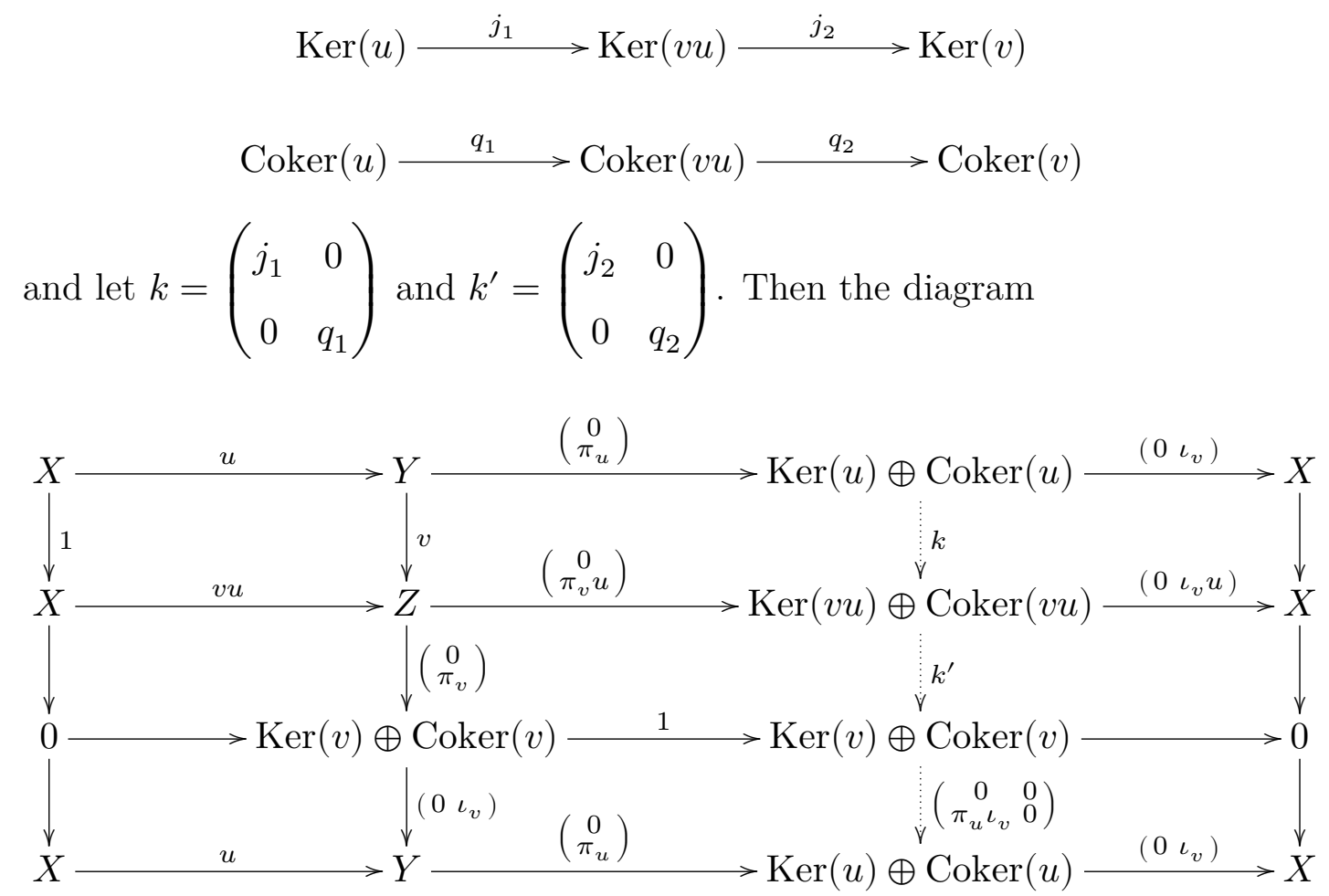

commutes and every row and column is a distinguished triangle.

\subsection{Chain Complexes}

Definition 1.2.1. An $R$-Chain Complex $C$ is a sequence

$$
C: \cdots \stackrel{d_{n+2}^{C}}{\longrightarrow} C_{n+1} \stackrel{d_{n+1}^{C}}{\longrightarrow} C_{n} \stackrel{d_{n}^{C}}{\longrightarrow} C_{n-1} \stackrel{d_{n-1}^{C}}{\longrightarrow} \cdots
$$

of $R$-modules $C_{n}$ and $R$-module homomorphisms $d_{n}^{C}: C_{n} \rightarrow C_{n-1}$ such that $d_{n}^{C}$ 。 $d_{n+1}^{C}=0$ for all $n$. If $C, D$ are $R$-complexes and $\psi_{n}: C_{n} \rightarrow D_{n}$ is a family of $R$ module homomorphisms such that $\psi_{n} \circ d_{n}^{D}=d_{n}^{C} \circ \psi_{n-1}$, then we call $\psi$ a morphism of chain complexes. 
The condition $d_{n}^{C} \circ d_{n+1}^{C}=0$ is equivalent to $\operatorname{Im} d_{n+1}^{C} \subseteq \operatorname{ker} d_{n}^{C}$. Therefore we can consider the quotient module $H_{n}(C)=\operatorname{ker} d_{n}^{C} / \operatorname{Im} d_{n+1}^{C}$ which we refer to as the nth Homology of $C$. We call $C$ exact (or acylic) if $\operatorname{Im} d_{n+1}^{C}=\operatorname{ker} d_{n}^{C}$ for all $n$, i.e. $H_{n}(C)=0$ for all $n$. The complex $C$ is called totally acyclic if each $C_{n}$ is a finitely generated free $R$-module, $C$ is exact, and $\operatorname{Hom}_{R}(C, R)$ is exact, i.e.

$$
\mathrm{H}(C)=0=\mathrm{H}\left(\operatorname{Hom}_{R}(C, R)\right) .
$$

Definition 1.2.2. A bi-complex $C$ is a family $\left\{C_{i, j}\right\}$ of $R$-modules together with maps

$$
d_{p, q}^{h}: C_{p, q} \rightarrow C_{p-1, q} \quad \text { and } \quad d_{p, q}^{v}: C_{p, q} \rightarrow C_{p, q-1}
$$

such that $d^{h} \circ d^{h}=d^{v} \circ d^{v}=d^{v} d^{h}+d^{h} d^{v}=0$. We can view the bi-complex $C$ via the diagram

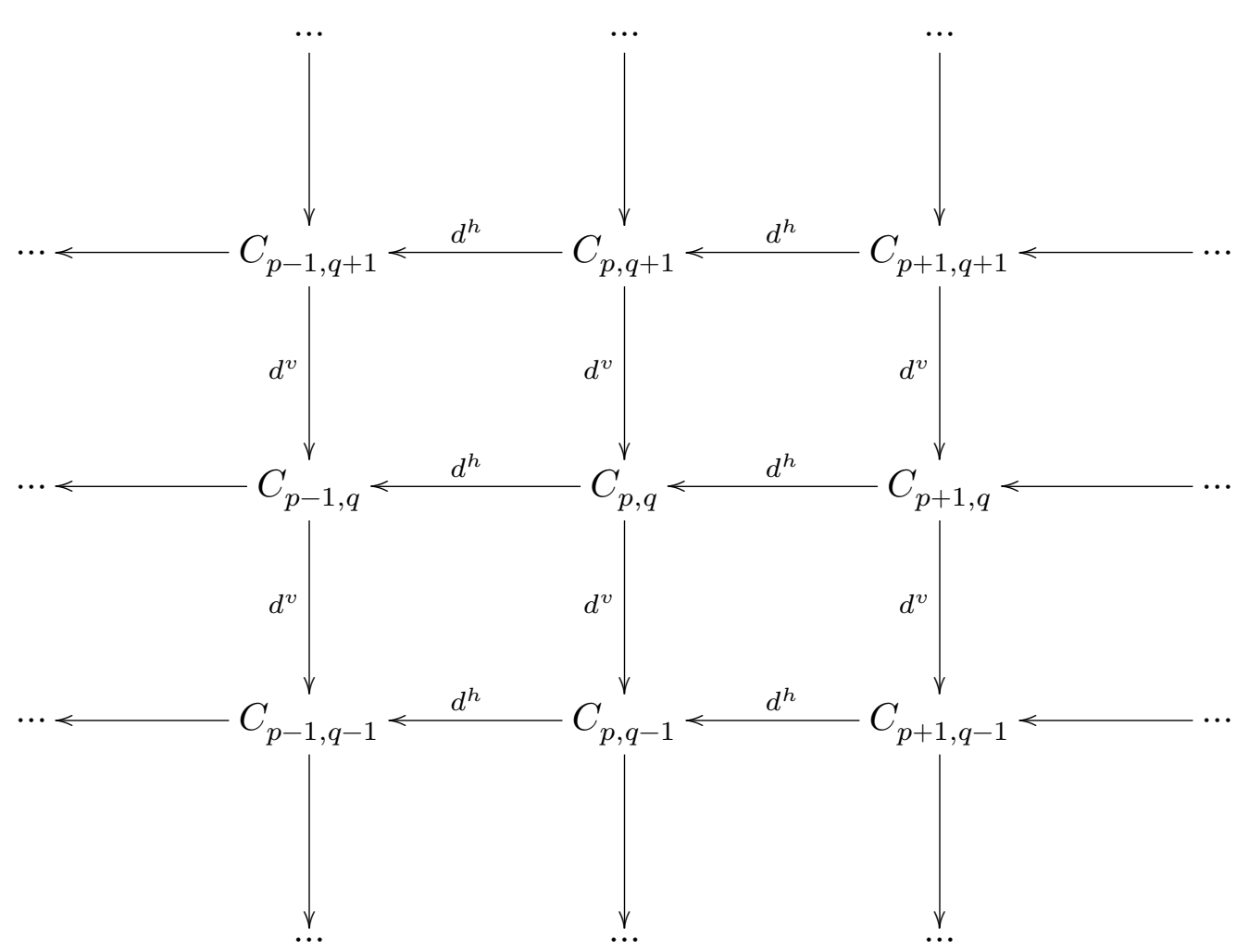

where each square anti-commutes. Notice that each row and each column is a chain complex. 
Definition 1.2.3. Let $C$ be a bi-complex of $R$-modules. The total complex of $C$, $\operatorname{Tot}^{\oplus}(C)$, is the complex given by

$$
\operatorname{Tot}^{\oplus}(C)_{n}=\bigoplus_{p+q=n} C_{p, q} \quad \text { and } \quad d_{n}^{\operatorname{Tot}^{\oplus}(C)}=\sum_{p+q=n} d_{p, q}^{h}+d_{p, q}^{v}
$$

The relation $d^{v} d^{h}+d^{h} d^{v}=0$ ensures that $d^{\operatorname{Tot}^{\oplus}(C)} \circ d^{\operatorname{Tot}^{\oplus}(C)}=0$.

Theorem 1.2.4. Acyclic Assembly Lemma (Weibel [22, 2.7.3]) Let $C$ be a bicomplex of $R$-modules. Then $\operatorname{Tot}^{\oplus}(C)$ is an acyclic chain complex assuming either of the following hold:

- $C$ is an upper half-plane complex with exact rows

- $C$ is a right half-plane complex with exact columns

Definition 1.2.5. Let $C$ and $D$ be chain complexes of right and left $R$-modules respectively. Consider the bi-complex $C \otimes_{R} D=\left\{C_{p} \otimes_{R} D_{q}\right\}$ with differentials given by $d_{p, q}^{h}=\Delta_{p, q}^{C}=d_{p}^{C} \otimes_{R} D_{q}$ and $d_{p, q}^{v}=\Delta_{p, q}^{D}=(-1)^{p-q} C_{p} \otimes d_{q}^{D}$. We call $C \otimes_{R} D$ the tensor product bi-complex of $C$ and $D$. Notice that the sign convention ensures that $\Delta^{C} \Delta^{D}+\Delta^{D} \Delta^{C}=0$.

We can now take the total complex $\operatorname{Tot}^{\oplus}\left(C \otimes_{R} D\right)$, called the (total) tensor product chain complex of $C$ and $D$. This complex has differentials given by

$$
d_{n}^{\operatorname{Tot}^{\oplus}\left(C \otimes_{R} D\right)}=\sum_{p+q=n} \Delta_{p, q}^{C}+\Delta_{p, q}^{D}
$$

In chapter 4 , we will take advantage of the tensor product chain complex to create interesting examples of exact complexes. Thus we need to know under what circumstances $\operatorname{Tot}^{\oplus}\left(C \otimes_{R} D\right)$ is exact.

Definition 1.2.6. A functor $F$ from the category of right $R$-modules to itself is called right exact if for any exact sequence of $R$-modules,

$$
X \rightarrow Y \rightarrow Z \rightarrow 0
$$


the sequence

$$
F(X) \rightarrow F(Y) \rightarrow F(Z) \rightarrow 0
$$

is also exact. The functor $F$ is called left exact if for the exact sequence

$$
0 \rightarrow X \rightarrow Y \rightarrow Z
$$

the sequence

$$
0 \rightarrow F(X) \rightarrow F(Y) \rightarrow F(Z)
$$

is also exact. We call $F$ exact if it is both left exact and right exact.

Proposition 1.2.7. (Rotman [20, 2.78]) For any right $R$-module $A$, the functor $A \otimes_{R}$ is right exact.

For a generic right $R$-module $A$, the functor $A \otimes_{R}$ - need not be left exact. If $A \otimes_{R}$ _ is left exact (and hence exact), then we refer to $A$ as flat.

Proposition 1.2.8. (Rotman $[20,3.46])$ If a right $R$-module $P$ is projective, then $P$ is flat.

Notice that if we let $C$ be an exact complex and let $D$ be a complex of flat modules that is bounded below, i.e. $D_{i}=0$ for all $i \ll 0$, then the acyclic assembly lemma implies that $\operatorname{Tot}^{\oplus}\left(C \otimes_{R} D\right)$ is an exact complex.

\subsection{The Triangulated Category $\mathrm{K}_{\mathrm{tac}}(R)$}

Homotopy Equivalence

Let $R$ be an associative ring, $C$ and $D$ be chain complexes of $R$-modules, and $\psi, \psi^{\prime}: C \rightarrow D$ be chain maps. We say $\psi$ is homotopic to $\psi^{\prime}$ if there exist maps 
$\lambda_{n}: C_{n} \rightarrow D_{n+1}$ such that $\psi_{n}-\psi_{n}^{\prime}=d_{n+1}^{D} \lambda_{n}+\lambda_{n-1} d_{n}^{C}$ and we write $\psi \sim \psi^{\prime}$. We refer to the $\lambda_{n}$ 's as homotopy maps.

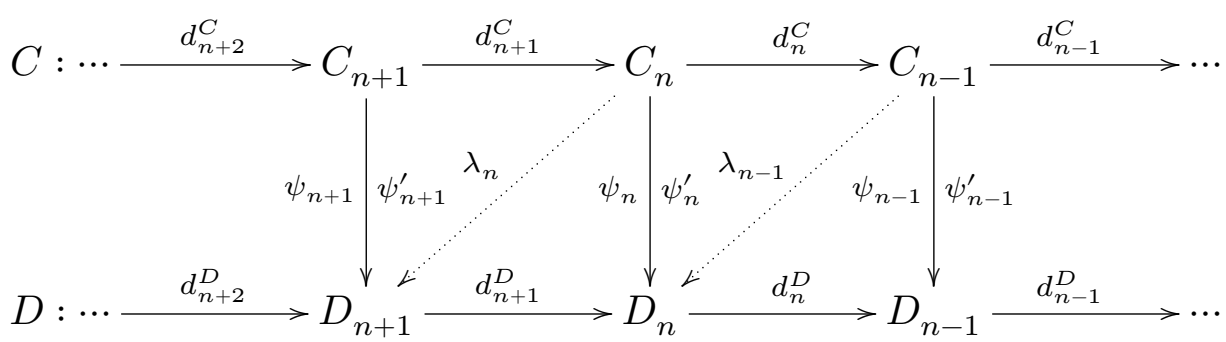

If there exist maps $\psi: C \rightarrow D$ and $\zeta: D \rightarrow C$ such that $\psi \circ \zeta \sim \operatorname{Id}_{D}$ and $\zeta \circ \psi \sim \operatorname{Id}_{C}$, then we say $C$ and $D$ are homotopically equivalent and we write $C \simeq D$. We denote by $\operatorname{Hom}_{R}(C, D)$ the set of homotopy equivalence classes of degree zero chain maps from $C$ to $D$.

The term homotopy map is inherited from algebraic topology. Notice that homotopy equivalence is a much weaker notion of equivalence than isomorphism. We are particularly interested in a category of homotopy equivalence classes since the construction of Eisenbud operators described in chapter 3 is only well defined up to homotopy.

Resolutions

Let $M$ be an $R$-module. An exact $R$-complex $P$ of the form

$$
P: \cdots \longrightarrow P_{2} \longrightarrow P_{1} \longrightarrow P_{0} \longrightarrow 0
$$

where each $P_{i}$ is a projective $R$-module is called a projective resolution of $M$. A Complete Resolution of $M$ is any totally acyclic chain complex $C$ such that the truncated complex

$$
C_{>i}: \cdots \longrightarrow C_{i+2} \longrightarrow C_{i+1} \longrightarrow 0
$$


is homotopically equivalent to

$$
P_{>i}: \cdots \longrightarrow P_{i+2} \longrightarrow P_{i+1} \longrightarrow 0
$$

for some $i$.

Lemma 1.3.1. Comparison Theorem (Rotman [20,6.16]) Let $M, N$ be $R$-modules and $f: M \rightarrow N$ be a homomorphism. Consider the diagram

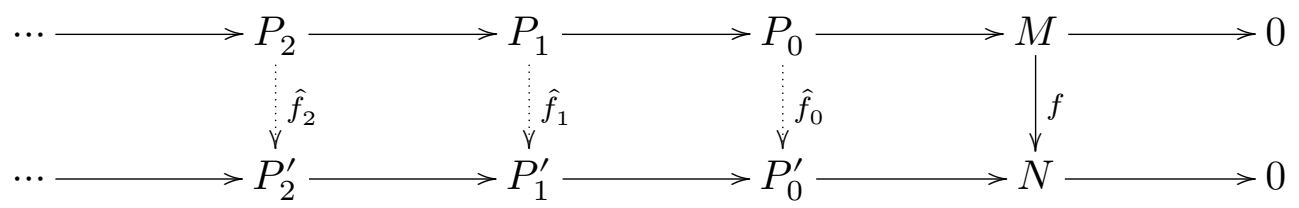

where the rows are complexes. If each $P_{n}$ in the top row is projective, and if the bottom row is exact, then there exists a chain map $\hat{f}: P \rightarrow P^{\prime}$ making the diagram commute. Moreover, any two such chain maps are homotopic.

If we let $P^{\prime}$ be a projective resolution of a module $N$ and let $\overline{\rho_{0}}: M \rightarrow N$ and $\overline{\mu_{0}}: N \rightarrow M$ be homomorphisms, then the comparison theorem implies that there exist chain maps $\rho: P \rightarrow P^{\prime}$ and $\mu: P^{\prime} \rightarrow P$. Furthermore, any chain map from $P$ to $P^{\prime}$ lifting $\overline{\rho_{0}}$ is homotopic to $\rho$ and any chain map from $P^{\prime}$ to $P$ lifting $\overline{\mu_{0}}$ is homotopic to $\mu$.

Proposition 1.3.2. (Avramov-Martsinkovsky [5, 5.3]) Complete resolutions are unique up to homotopy equivalence.

The following is an alternate proof to that given by Avramov and Martsinkovsky.

Proof. If $C, C^{\prime}$ are complete resolutions of $M, N$ respectively, then $C_{>i} \simeq P_{>i}$ and $C_{>k}^{\prime} \simeq P_{>k}^{\prime}$ for some $i, k$. Thus there exist maps $h_{>i}: C_{>i} \rightarrow P_{>i}, h_{>k}^{\prime}: C_{>k}^{\prime} \rightarrow P_{>k}^{\prime}$, $j_{>i}: P_{>i} \rightarrow C_{>i}$, and $j_{>k}^{\prime}: P_{>k}^{\prime} \rightarrow C_{>k}^{\prime}$ such that $h_{>i} \circ j_{>i} \sim \operatorname{Id}_{P_{>i}}, j_{>i} \circ h_{>i} \sim \operatorname{Id}_{C_{>i}}$, $h_{>k}^{\prime} \circ j_{>k}^{\prime} \sim \operatorname{Id}_{P_{>k}^{\prime}}$, and $j_{>k}^{\prime} \circ h_{>k}^{\prime} \sim \operatorname{Id}_{C_{>k}^{\prime}}$.

We can set $l=\max (i, k)$ so that $C_{>l} \simeq P_{>l}$ and $C_{>l}^{\prime} \simeq P_{>l}^{\prime}$. Hence for any homomorphisms $\overline{\rho_{0}}: M \rightarrow N$ and $\overline{\mu_{0}}: N \rightarrow M$ with $\rho: P \rightarrow P^{\prime}$ and $\mu: P^{\prime} \rightarrow P$ 
as above, there exist chain maps $\gamma_{>l}: C_{>l} \rightarrow C_{>l}^{\prime}$ and $\delta_{>l}: C_{>l}^{\prime} \rightarrow C_{>l}$ where $\gamma_{n}=j_{n}^{\prime} \circ \rho_{n} \circ h_{n}$ and $\delta_{n}=j_{n} \circ \mu_{n} \circ h_{n}^{\prime}$ for $n \geq l$. Now consider the following sequence.

$$
\cdots \rightarrow \operatorname{Hom}\left(C_{l}, C_{l}^{\prime}\right) \rightarrow \operatorname{Hom}\left(C_{l+1}, C_{l}^{\prime}\right) \rightarrow \operatorname{Hom}\left(C_{l+2}, C_{l}^{\prime}\right) \rightarrow \cdots
$$

This sequence is exact since $C$ is totally acyclic. Now consider the following diagram.

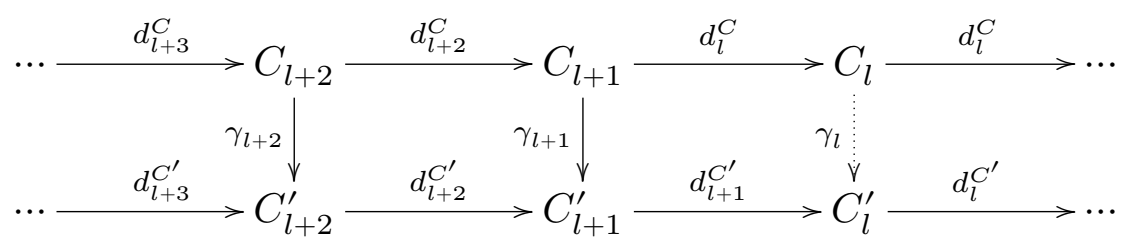

We would like to show that $\gamma_{n}$ exists for $n \leq l$ such that the diagram commutes. We know that the diagram commutes for $C_{>l}$, thus

$$
d_{l+1}^{C^{\prime}} \circ \gamma_{l+1} \circ d_{l+2}^{C}=d_{l+1}^{C^{\prime}} \circ d_{l+2}^{C^{\prime}} \circ \gamma_{l+2}=0
$$

Therefore we have

$$
d_{l+1}^{C^{\prime}} \circ \gamma_{l+1} \in \operatorname{ker}\left(\operatorname{Hom}\left(d_{l+2}^{C}, C_{l}^{\prime}\right)\right)=\operatorname{Im}\left(\operatorname{Hom}\left(d_{l+1}^{C}, C_{l}^{\prime}\right)\right)
$$

Then there exists $\gamma_{l} \in \operatorname{Hom}\left(C_{l}, C_{l}^{\prime}\right)$ such that $d_{l+1}^{C^{\prime}} \circ \gamma_{l+1}=\gamma_{l} \circ d_{l+1}^{C}$. We can now apply induction to construct $\gamma_{n}: C_{n} \rightarrow C_{n}^{\prime}$ for $n \leq l$ such that $d_{n+1}^{C^{\prime}} \circ \gamma_{n+1}=\gamma_{n} \circ d_{n+1}^{C}$ and similarly for $\delta_{n}: C_{n}^{\prime} \rightarrow C_{n}$.

Thus we now have chain maps $\gamma: C \rightarrow C^{\prime}$ and $\delta: C^{\prime} \rightarrow C$. If $M=N$ and $\rho_{0}=\mu_{0}=\operatorname{Id}_{M}$, then $\rho \circ \mu \sim \operatorname{Id}_{p^{\prime}}$ and $\mu \circ \rho \sim \operatorname{Id}_{P}$. Therefore $\gamma \circ \delta \sim \operatorname{Id}_{C}$ and $\delta \circ \gamma \sim \operatorname{Id}_{C^{\prime}}$, hence $C \simeq C^{\prime}$.

\section{The Homotopy Category}

Consider the category of homotopy equivalence classes of chain complexes, $\mathrm{K}(R)$, with distinguished triangles of the form

$$
C \stackrel{t}{\longrightarrow} D \longrightarrow \text { Cone }(t) \longrightarrow \Sigma C
$$


where $t$ is a zero degree chain map from $C$ to $D$. The shift functor $\Sigma$ simply moves every module in the complex to the left, i.e $\left(\Sigma^{i} C\right)_{n}=C_{n-i}$. For simplicity we will write $\Sigma C=\Sigma^{1} C$. The differentials of the shifted complex are defined as $d_{n}^{\Sigma^{i} C}=$ $(-1)^{i}\left(\sigma_{C}^{i}\right)_{n-i-1} d_{n-i}^{C}\left(\sigma_{C}^{i}\right)_{n}^{-1}$ where $\sigma_{C}^{i}: C \rightarrow \Sigma^{i} C$ is the natural isomorphism. Notice that $\sigma_{\Sigma^{i} C}^{-i}=\left(\sigma_{C}^{i}\right)^{-1}$.

Remark 1.3.3. We include the $\sigma$ maps for completeness sake. However, the reader may wish to ignore these maps as well as the shift indices on the differentials on a first reading in order to more easily understand the main thrust of the arguments.

The Mapping Cone of $t$ is the chain complex Cone $(t)=\Sigma C \oplus D$ with differentials given by

$$
d_{n}^{\operatorname{Cone}(t)}=\left(\begin{array}{cc}
d_{n}^{\Sigma C} & 0 \\
t_{n-1}\left(\sigma_{C}^{1}\right)_{n-1}^{-1} & d_{n}^{D}
\end{array}\right) .
$$

Proposition 1.3.4. (Weibel $[22,10.2 .4]) \mathrm{K}(R)$ is a triangulated category.

We will now restrict our view to the subcategory of totally acyclic complexes, $\mathrm{K}_{\mathrm{tac}}(R)$.

Proposition 1.3.5. $\mathrm{K}_{\mathrm{tac}}(R)$ is a triangulated subcategory of $\mathrm{K}(R)$.

Proof. The shift of any totally acyclic complex is clearly totally acyclic. Thus it only remains to show that for any totally acyclic complexes $C, D$ and any chain map $t: C \rightarrow D$, Cone $(t)$ is totally acyclic.

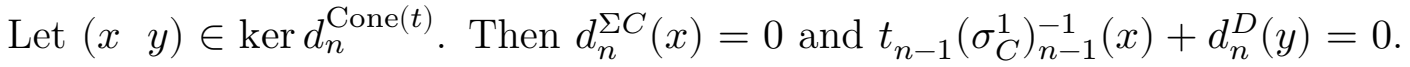
Then $x \in \operatorname{Ker} d_{n}^{\Sigma C}=\operatorname{Im} d_{n+1}^{\Sigma C}$. So there exists $z \in(\Sigma C)_{n+1}$ such that $d_{n+1}^{\Sigma C}(z)=x$.

Since $t$ is a chain map, we know that $-t_{n}\left(\sigma_{C}^{1}\right)^{-1} d_{n+1}^{\Sigma C}-d_{n}^{D} t_{n}\left(\sigma_{C}^{1}\right)^{-1}=0$. Thus $0=-t_{n}\left(\sigma_{C}^{1}\right)^{-1} d_{n+1}^{\Sigma C}(z)-d_{n}^{D} t_{n}\left(\sigma_{C}^{1}\right)^{-1}(z)=-t_{n}\left(\sigma_{C}^{1}\right)^{-1}(x)-d_{n}^{D} t_{n}\left(\sigma_{C}^{1}\right)^{-1}(z)=d_{n}^{D}(y)-$ $d_{n}^{D} t_{n}\left(\sigma_{C}^{1}\right)^{-1}(z)$. Hence $y-t_{n}\left(\sigma_{C}^{1}\right)^{-1}(z) \in \operatorname{ker} d_{n}^{D}=\operatorname{Im} d_{n+1}^{D}$. So there exists $w \in$ $D_{n+1}$ such that $d_{n+1}^{D}(w)=y-t_{n}\left(\sigma_{C}^{1}\right)^{-1}(z)$. Therefore $d_{n+1}^{\mathrm{Cone}(t)}(z w)=(x y)$ and so $\left(\begin{array}{ll}x & y\end{array}\right) \in \operatorname{Im} d^{\operatorname{Cone}(t)_{n+1}}$. Thus Cone $(t)$ is exact. 
We can similarly show that $\operatorname{Hom}(\operatorname{Cone}(t), R)$ is exact by using the facts that $\operatorname{Hom}(C, R)$ and $\operatorname{Hom}(D, R)$ are exact. 


\section{Chapter 2}

\section{Support and Rank Varieties in $\mathrm{K}_{\mathrm{tac}}(R)$}

In this chapter we will define the support variety and rank variety for a pair of totally acyclic chain complexes. We will first describe the ring of cohomology operators, $R\left[\chi_{1}, \ldots, \chi_{c}\right]$, defined by a pair of complexes $C, D \in \mathrm{K}_{\mathrm{tac}}(R)$. To do this we use Eisenbud's [15] construction of degree 2 chain maps to create an $R\left[\chi_{1}, \ldots, \chi_{c}\right]$ action on the graded module $\bigoplus_{i \in \mathbb{Z}} \operatorname{Hom}_{R}(C, D)$. We also show that the action of these operators is well defined.

The support variety is defined as the zero set of the annihilator of this ring of cohomology operators. The rank variety is defined as the set of points $\bar{a} \in k^{c}$ such that $\operatorname{Hom}_{Q_{a}}$ vanishes in high degree where $Q_{a}$ is a hypersurface ring. However since $C, D$ are in general not $Q_{a}$-complexes, we will also need to take advantage of a pair of triangulated functors to move between $\mathrm{K}_{\mathrm{tac}}(R)$ and $\mathrm{K}_{\mathrm{tac}}\left(Q_{a}\right)$.

\subsection{Eisenbud and Cohomology Operators in $\mathrm{K}_{\text {tac }}(R)$}

From this point forward we will let $R=Q /\left(f_{1}, \ldots, f_{c}\right)$ where $(Q, m, \mathbb{k})$ is a local ring and $f=f_{1}, \ldots, f_{c}$ is a regular sequence in $m^{2}$. We will also assume that the residue field $\mathbb{k}$ is algebraically closed. For pairs of modules over a complete intersection ring, there are multiple equivalent methods of constructing cohomology operators. To construct similar operators in $\mathrm{K}_{\mathrm{tac}}(R)$, we will use Eisenbud's [15] method of lifting chain complex differentials to create degree 2 chain maps. 
Eisenbud's Construction

Given an $R$-complex $C$, we define $\widetilde{C}$ to be a sequence of $Q$-free module homomorphisms such that

$$
\widetilde{C} \otimes_{Q} R \simeq C
$$

and we call $\widetilde{C}$ a lift of $C$. Note that $\widetilde{C}$ is not necessarily a chain complex. In particular,

$$
\tilde{d}_{n-1}^{C} \circ \tilde{d}_{n}^{C}=\sum_{k=1}^{c} f_{k} \tilde{t}_{k, n}
$$

where $\tilde{t}_{k, n}:{\widetilde{C_{n}}}_{n}{\widetilde{C_{n-2}}}_{n}$. We will write $\tilde{t}_{k}$ for the family of maps $\left\{\tilde{t}_{k, n}\right\}_{n \in \mathbb{Z}}$.

Proposition 2.1.1. (Eisenbud [15, 1.1]) The map $t_{k}=\sigma_{C}^{2}\left(\tilde{t}_{k} \otimes_{Q} R\right): C \rightarrow \Sigma^{2} C$ is a morphism.

Now let $D$ be another chain complex with

$$
\tilde{d}_{n-1}^{D} \circ \tilde{d}_{n}^{D}=\sum_{k=1}^{c} f_{k} \tilde{s}_{k, n} \text { and } s_{k}=\sigma_{D}^{2}\left(\tilde{s}_{k} \otimes_{Q} R\right) .
$$

Proposition 2.1.2. (Eisenbud $[15,1.3]$ ) If $\psi: C \rightarrow D$ is a chain map, then

$$
\psi \circ\left(\sigma_{C}^{2}\right)^{-1} \circ t_{k} \sim\left(\sigma_{D}^{2}\right)^{-1} \circ s_{k} \circ \psi
$$

We will now give a reorganized and more detailed version of Eisenbud's original proof.

Proof. Since $\psi$ is an $R$-complex chain map, lifting it to $Q$ yields

$$
\widetilde{\psi} \tilde{d}^{C}-\tilde{d}^{D} \widetilde{\psi}=\sum_{j=1}^{c} f_{j} \tilde{\tau}_{j}
$$

We can also lift the maps $t_{k}$ and $s_{k}$ to $Q$ and use the facts that $\left(\tilde{d}^{C}\right)^{2}=\sum_{j=1}^{c} f_{j} \tilde{t}_{j}$ and $\left(\tilde{d}^{D}\right)^{2}=\sum_{j=1}^{c} f_{j} \tilde{s}_{j}$ to get

$$
f_{k}\left(\tilde{\psi}_{k}-\tilde{s}_{k} \widetilde{\psi}\right)=\widetilde{\psi}\left(\left(\tilde{d}^{C}\right)^{2}-\sum_{j \neq k} f_{j} \tilde{t}_{j}\right)-\left(\left(\tilde{d}^{D}\right)^{2}-\sum_{j \neq k} f_{j} \tilde{s}_{j}\right) \widetilde{\psi}
$$


We will now commute the $\widetilde{\psi}$ with one copy each of $\tilde{d}^{C}$ and $\tilde{d}^{D}$ and subtract like terms to get

$$
f_{k}\left(\widetilde{\psi}_{k}-\tilde{s}_{k} \widetilde{\psi}\right)=\sum_{j \neq k} f_{j} \widetilde{\psi}\left(\tilde{s}_{j}-\tilde{t}_{j}\right)+\sum_{j=1}^{c} f_{j}\left(\tilde{d}^{D} \tilde{\tau}_{j}+\tilde{\tau}_{j} \tilde{d}^{C}\right) .
$$

Taking $f_{k}\left(\tilde{d}^{D} \tilde{\tau}_{k}+\tilde{\tau}_{k} \tilde{d}^{C}\right)$ to the left yields

$$
\begin{gathered}
f_{k}\left(\widetilde{\psi} \widetilde{t_{k}}-\widetilde{s}_{k} \widetilde{\psi}-\left(\tilde{d}^{D} \tilde{\tau}_{k}+\tilde{\tau}_{k} \tilde{d}^{C}\right)\right)= \\
\sum_{j \neq k} f_{j} \widetilde{\psi}\left(\tilde{s}_{j}-\tilde{t}_{j}\right)+\sum_{j \neq k} f_{j}\left(\tilde{d}^{D} \tilde{\tau}_{j}+\tilde{\tau}_{j} \tilde{d}^{C}\right) .
\end{gathered}
$$

Thus we see that

$$
\operatorname{Im}\left(\widetilde{\psi} \tilde{t}_{k}-\tilde{s}_{k} \widetilde{\psi}-\left(\tilde{d}^{D} \tilde{\tau}_{k}+\tilde{\tau}_{k} \tilde{d}^{C}\right)\right) \subseteq\left(f_{1}, \ldots, f_{c}\right) \Sigma^{i} D
$$

and so tensoring down to $R$ we have

$$
\psi\left(\sigma_{C}^{2}\right)^{-1} t_{k}-\left(\sigma_{D}^{2}\right)^{-1} s_{k} \psi=d^{D} \tau_{k}+\tau_{k} d^{C}
$$

We can consider the map $\tau_{k}$ as a homotopy map so that $\psi\left(\sigma_{C}^{2}\right)^{-1} t_{k}-\left(\sigma_{D}^{2}\right)^{-1} s_{k} \psi \sim 0$ as desired.

The next two corollaries show that the $t_{k}$ 's are well defined in $\mathrm{K}_{\text {tac }}(R)$. Corollary 2.1.3. (Eisenbud $[15,1.4,1.5]$ ) The $t_{k}$ 's are uniquely determined up to homotopy and $t_{k} t_{j} \sim t_{j} t_{k}$.

Corollary 2.1.4. If $C$ and $D$ are homotopically equivalent with $\psi: C \rightarrow D$ and $\zeta: D \rightarrow C$ such that $\psi \zeta \sim \operatorname{Id}_{D}$ and $\zeta \psi \sim \operatorname{Id}_{C}$, then $t_{k} \sim \zeta s_{k} \psi$ and $s_{k} \sim \psi t_{k} \zeta$.

Proof. By Proposition 2.1.2, $\psi t_{k} \sim s_{k} \psi$ and thus $t_{k} \sim \zeta \psi t_{k} \sim \zeta s_{k} \psi$. Similarly $s_{k} \sim \psi t_{k} \zeta$

We will now consider the maps $\operatorname{Hom}_{R}\left(t_{k}, D\right)$, which we will call Eisenbud operators. The Eisenbud operators define an action on the set $\bigoplus_{i \in \mathbb{Z}} \operatorname{Hom}_{R}\left(C, \Sigma^{i} D\right)$ via 
composition. We can also define operators $\operatorname{Hom}_{R}\left(C, s_{k}\right)$. We would like to show that the action of $\operatorname{Hom}_{R}\left(t_{k}, D\right)$ agrees with the action of $\operatorname{Hom}_{R}\left(C, s_{k}\right)$.

Corollary 2.1.5. Consider the graded R-module maps

$$
\begin{gathered}
w_{k}: \bigoplus_{i \in \mathbb{Z}} \operatorname{Hom}_{R}\left(\Sigma^{-i} C, D\right) \rightarrow \bigoplus_{i \in \mathbb{Z}} \operatorname{Hom}_{R}\left(\Sigma^{-i} C, D\right), \\
v_{k}: \bigoplus_{i \in \mathbb{Z}} \operatorname{Hom}_{R}\left(C, \Sigma^{i} D\right) \rightarrow \bigoplus_{i \in \mathbb{Z}} \operatorname{Hom}_{R}\left(C, \Sigma^{i} D\right),
\end{gathered}
$$

and

$$
\phi: \bigoplus_{i \in \mathbb{Z}} \operatorname{Hom}_{R}\left(\Sigma^{-i} C, D\right) \rightarrow \bigoplus_{i \in \mathbb{Z}} \operatorname{Hom}_{R}\left(C, \Sigma^{i} D\right)
$$

defined by

$$
\begin{gathered}
\left(w_{k}\right)_{j}=\operatorname{Hom}_{R}\left(\left(\sigma_{C}^{-j+2}\right)^{-1}, D\right) \circ \operatorname{Hom}_{R}\left(t_{k}, D\right) \circ \operatorname{Hom}_{R}\left(\sigma_{\Sigma^{2} C}^{-j-2}, D\right), \\
\left(v_{k}\right)_{j}=\operatorname{Hom}_{R}\left(C, \sigma_{\Sigma^{2} D}^{j-2}\right) \circ \operatorname{Hom}_{R}\left(C, s_{k}\right) \circ \operatorname{Hom}_{R}\left(C,\left(\sigma_{D}^{j}\right)^{-1}\right),
\end{gathered}
$$

and

$$
\phi_{j}(\alpha)=\sigma_{D}^{j} \circ \alpha \circ \sigma_{C}^{-j} \text { for any } \alpha \in \operatorname{Hom}_{R}\left(\Sigma^{-j} C, D\right) .
$$

The following diagram commutes up to homotopy.

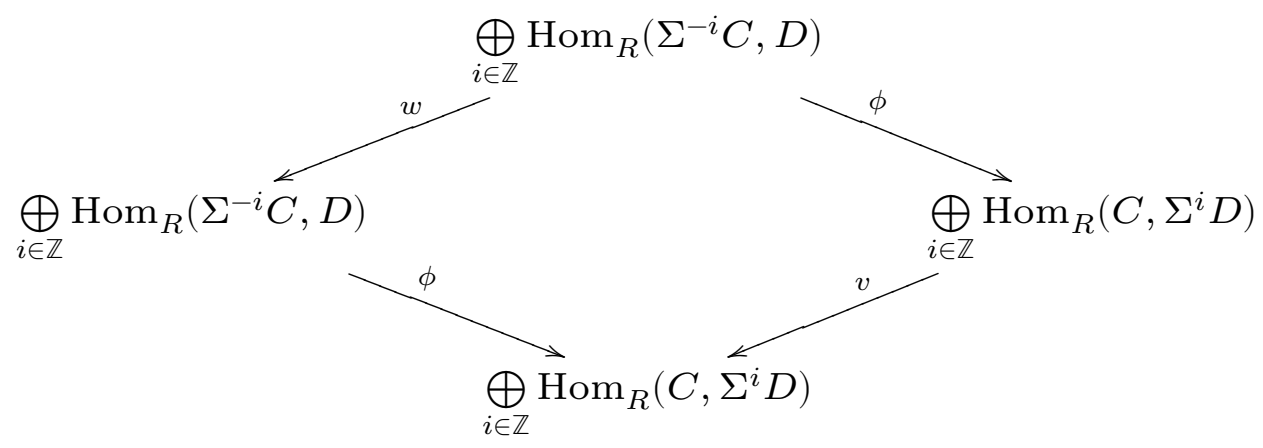

Proof. Let $\alpha \in \operatorname{Hom}_{R}\left(\Sigma^{-j} C, D\right)$. Proposition 2.1.2 implies that

$$
\sigma_{\Sigma^{2} D}^{j-2} \circ s_{k} \circ\left(\sigma_{D}^{j}\right)^{-1} \circ \phi(\alpha) \sim \phi(\alpha) \circ \sigma_{\Sigma^{2} C}^{-j-2} \circ t_{k} \circ\left(\sigma_{C}^{-j+2}\right)^{-1} .
$$


The Ring of Cohomology Operators

Now consider the diagram

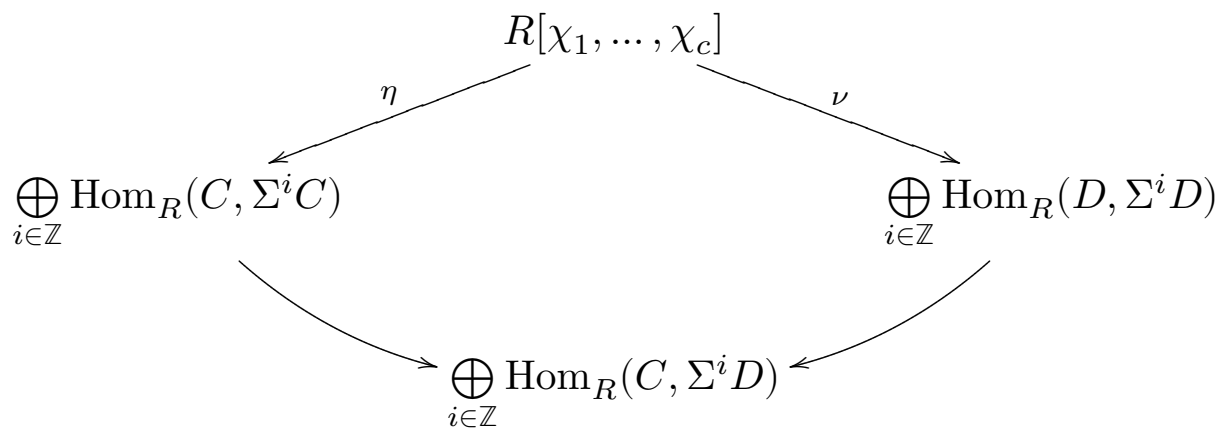

where $\eta\left(\chi_{k}\right)=t_{k}$ and $\nu\left(\chi_{k}\right)=s_{k}$. We can regard $\bigoplus_{i \in \mathbb{Z}} \operatorname{Hom}_{R}\left(C, \Sigma^{i} D\right)$ as a left $\bigoplus_{i \in \mathbb{Z}} \operatorname{Hom}_{R}\left(D, \Sigma^{i} D\right)$ right $\bigoplus_{i \in \mathbb{Z}} \operatorname{Hom}_{R}\left(C, \Sigma^{i} C\right)$ graded bimodule with the actions defined by composition. Then $\bigoplus_{i \in \mathbb{Z}} \operatorname{Hom}_{R}\left(C, \Sigma^{i} D\right)$ is an $R\left[\chi_{1}, \ldots, \chi_{c}\right]$-module with the action $\chi_{k} \cdot \alpha=w(\alpha)$ for any $\alpha \in \bigoplus_{i \in \mathbb{Z}} \operatorname{Hom}_{R}\left(C, \Sigma^{i} D\right)$. The grading is given by $\left|\chi_{k}\right|=2$ for all $k$ and $|r|=0$ for all $r \in R$. We refer to the $\chi_{k}$ 's as cohomology operators and $R\left[\chi_{1}, \ldots, \chi_{c}\right]$ as the ring of cohomology operators.

Corollary 2.1.5 implies that

$$
\chi_{k} \cdot \alpha=w_{k}(\alpha) \sim \phi \circ v_{k} \circ \phi^{-1}(\alpha)
$$

and so the action of $R\left[\chi_{1}, \ldots, \chi_{c}\right]$ on $\bigoplus_{i \in \mathbb{Z}} \operatorname{Hom}_{R}\left(C, \Sigma^{i} D\right)$ is independent of the choice of using $C$ or $D$ for the cohomology operators. We would furthermore like this construction to be independent of the choice of basis of $\left(f_{1}, \ldots, f_{c}\right)$.

Proposition 2.1.6. (Eisenbud $[15,1.7]$ ) Let $Q, Q^{\prime}$ be local rings and let $f_{1}, \ldots, f_{c}$ and $f_{1}^{\prime}, \ldots, f_{c^{\prime}}^{\prime}$ be regular sequences in $Q, Q^{\prime}$ respectively. Consider the complete intersection rings $R=Q /\left(f_{1}, \ldots, f_{c}\right)$ and $R^{\prime}=Q^{\prime} /\left(f_{1}^{\prime}, \ldots, f_{c^{\prime}}^{\prime}\right)$. Let $\alpha: Q \rightarrow Q^{\prime}$ be a ring homomorphism such that $\alpha\left(\left(f_{1}, \ldots, f_{c}\right)\right) \subseteq\left(f_{1}^{\prime}, \ldots, f_{c^{\prime}}^{\prime}\right)$ with

$$
\alpha\left(f_{i}\right)=\sum_{j=1}^{c^{\prime}} a_{i j} f_{j}^{\prime} \text { for some elements } a_{i j} \in Q^{\prime} .
$$


Also let $C \in \mathrm{K}_{\mathrm{tac}}(R)$ with the family of maps $t_{k}: C \rightarrow \Sigma^{2} C$ as defined above. Consider the $R^{\prime}$-complex $R^{\prime} \otimes_{R} C$ with the family of maps $t_{j}^{\prime}$. Then $t_{j}^{\prime}$ is homotopic to $\sum_{k=1}^{c} a_{k j}\left(R^{\prime} \otimes_{R} t_{k}\right)$.

If we let $Q=Q^{\prime}$ and let $f_{1}, \ldots, f_{c}$ and $f_{1}^{\prime}, \ldots, f_{c}^{\prime}$ be two $Q$-regular sequences that generate the same ideal and we let $\alpha$ be the identity map on $R$, then the above proposition implies that $\sum_{k=1}^{c} a_{k j} t_{k} \sim t_{j}^{\prime}$ for some $a_{k j} \in Q$. Thus we have $\chi_{j}^{\prime} \cdot \alpha=$ $w_{j}(\alpha)=\sum_{k=1}^{c} a_{k j} w_{k}^{\prime}(\alpha)=\sum_{k=1}^{c} a_{k j} \chi_{k} \cdot \alpha$ for any chain map $\alpha \in \bigoplus_{i \in \mathbb{Z}} \operatorname{Hom}_{R}\left(C, \Sigma^{i} D\right)$, i.e. each $\chi_{j}^{\prime}$ is a linear combination of the $\chi_{k}$ 's.

\subsection{Definitions of Support and Rank Varieties}

We will now translate the definitions of support and rank varieties of modules to the category of totally acyclic complexes.

\section{Support Varieties}

Avramov and Buchweitz $[2,2.1]$ define the support variety $V(Q, f, M, N)$ of a pair of $R$-modules $M, N$ to be the zero set of the annihilator of the $\mathbb{k}\left[\chi_{1}, \ldots, \chi_{c}\right]$ module $E=\bigoplus_{i \geq 0} \operatorname{Ext}_{R}^{i}(M, N) \otimes_{R} \mathbb{k}$, i.e.

$$
\begin{gathered}
V(Q, f, M, N)=\left\{\left(b_{1}, \ldots, b_{c}\right) \in \mathbb{k}^{c} \mid \phi\left(b_{1}, \ldots, b_{n}\right)=0\right. \\
\text { for all } \phi \in \text { Ann } E\} \cup\{0\} .
\end{gathered}
$$

We will now give a similar definition for pairs of totally acyclic complexes by replacing $\bigoplus_{i \geq 0} \operatorname{Ext}_{R}^{i}(M, N)$ with the $R\left[\chi_{1}, \ldots, \chi_{c}\right]$-module $\underset{i \geq 0}{\bigoplus} \operatorname{Hom}_{R}\left(C, \Sigma^{i} D\right)$, a submodule of $\bigoplus_{i \in \mathbb{Z}} \operatorname{Hom}_{R}\left(C, \Sigma^{i} D\right)$ that we discussed in the previous section.

Definition 2.2.1. Let $R=Q /\left(f_{1}, \ldots, f_{c}\right)$ where $Q$ is a regular local ring with residue field $\mathbb{k}$ and $f_{1}, \ldots, f_{c}$ is a regular sequence. Also let $S=R\left[\chi_{1}, \ldots, \chi_{c}\right]$ be the ring of cohomology operators defined by $f_{1}, \ldots, f_{c}$. Then the support variety $V(Q, f, C, D)$ 
of a pair of complexes $C, D \in \mathrm{K}_{\mathrm{tac}}(R)$ is the zero set of the annihilator of the $S \otimes_{R} \mathbb{k}^{\mathrm{k}}$ module $E=\bigoplus_{i \geq 0} \operatorname{Hom}_{R}\left(C, \Sigma^{i} D\right) \otimes_{R} \mathbb{k}$. That is,

$$
\begin{gathered}
V(Q, f, C, D)=\left\{\left(b_{1}, \ldots, b_{c}\right) \in \mathbb{k}^{c} \mid \phi\left(b_{1}, \ldots, b_{n}\right)=0\right. \\
\text { for all } \phi \in \text { Ann } E\} \cup\{0\} .
\end{gathered}
$$

This definition is especially useful for computing examples by simply decomposing the differentials of $C$ to find the cohomology operators.

\section{Rank Varieties}

Carlson $[13,4.1]$ defined the rank variety $W(M)$ of a module $M$ over a group algebra $\mathbb{k} G \cong \mathbb{k}\left[\left[x_{1}, \ldots, x_{c}\right]\right] /\left(x_{1}^{p}, \ldots, x_{c}^{p}\right)$ of an elementary abelian $p$-group $G$, where $p=\operatorname{char}(\mathbb{k})$, to be the set

$$
W(M)=\left\{\bar{a} \in \mathbb{k}^{c} \mid M \downarrow_{\mathbb{k}\left[l_{a}\right]} \text { is not free }\right\} \cup\{0\}
$$

where $\bar{a}$ is the image of $a=\left(a_{1}, \ldots, a_{c}\right)$ with $a_{i} \in \mathbb{k} G$ and $l_{a}=a_{1} x_{1}+\cdots+a_{c} x_{c} \in \mathbb{k} G$. By a theorem of Avramov [1, 7.5], the projective dimension of a module $M$ over the ring $Q_{a}=\mathbb{k}\left[\left[x_{1}, \ldots, x_{c}\right]\right] /\left(l_{a}^{p}\right)$ is finite if and only if $M$ is free over $\mathbb{k}\left[l_{a}\right]$. Thus

$$
W(M)=\left\{\bar{a} \in \mathbb{k}^{c} \mid \operatorname{Ext}_{Q_{a}}^{i}(M, \mathbb{k}) \neq 0 \text { for infinitely many i }\right\} \cup\{0\} .
$$

Avramov and Buchweitz $[2,2.5]$ generalized this for a pair of modules $M, N$ over a complete intersection ring $R=Q /\left(f_{1}, \ldots, f_{c}\right)$ by setting

$$
W(Q, f, M, N)=\left\{\bar{a} \in \mathbb{k}^{c} \mid \operatorname{Ext}_{Q_{a}}^{i}(M, N) \neq 0 \text { for infinitely many i }\right\} \cup\{0\}
$$

where $a=\left(a_{1}, \ldots, a_{c}\right) \in Q^{c}$ denotes some lifting of $\bar{a}=\left(\overline{a_{1}}, \ldots, \overline{a_{c}}\right) \in \mathbb{k}^{c}$ and $Q_{a}=$ $Q / f_{a} Q$ with $f_{a}=a_{1} f_{1}+\cdots+a_{c} f_{c} \in Q$.

Remark 2.2.2. Avramov and Buchweitz referred to $W(Q, f, M, N)$ as the support variety after proving it is equivalent to $V(Q, f, M, N)$. 
We would like to give a similar definition for the rank variety of a pair of totally acyclic complexes $C, D$ in terms of the vanishing of $\operatorname{Hom}_{Q_{a}}$ where $Q_{a}$ is a complete intersection ring intermediate to $Q$ and $R$. However, totally acyclic $R$-complexes $C, D$ are not totally acyclic $Q_{a}$-complexes. The following pair of adjoint triangle functors by Bergh, Jorgensen, and Moore will allow us to go back and forth between $\mathrm{K}_{\mathrm{tac}}(R)$ and $\mathrm{K}_{\mathrm{tac}}\left(Q_{a}\right)$.

Definition 2.2.3. (Bergh-Jorgensen-Moore $[10,2.1]$ ) Let $R=Q / I$ where $Q$ is a Gorenstein ring and $I$ is an ideal with $\operatorname{proj}^{\operatorname{dim}_{Q}} R<\infty$, and let $C \in \mathrm{K}_{\text {tac }}(R)$. Then define $T: \mathrm{K}_{\mathrm{tac}}(R) \rightarrow \mathrm{K}_{\mathrm{tac}}(Q)$ by letting $T C \in \mathrm{K}_{\mathrm{tac}}(Q)$ be a complete resolution of $\operatorname{Im} d_{o}^{C}$ over $\mathrm{Q}$. Given a chain map $\alpha: C \rightarrow C^{\prime}$ in $\mathrm{K}_{\mathrm{tac}}(R)$, we have the map $u: \operatorname{Im}\left(d_{o}^{C}\right) \rightarrow \operatorname{Im}\left(d_{o}^{C^{\prime}}\right)$ induced by the map $\alpha$. Then $T \alpha: T C \rightarrow T C^{\prime}$ is the homotopy equivalence class of the comparison map $\tilde{u}: T C \rightarrow T C^{\prime}$. Define $S: \mathrm{K}_{\mathrm{tac}}(Q) \rightarrow \mathrm{K}_{\mathrm{tac}}(R)$ by $S D=D \otimes_{Q} R$ and $S \alpha=\alpha \otimes_{Q} R$.

Remark 2.2.4. Notice that $T$ is well defined because complete resolutions are unique up to homotopy equivalence (Proposition 1.3.2).

Theorem 2.2.5. (Bergh-Jorgensen-Moore [10,3.1]) The triangle functors $S$ and $T$ form an adjoint pair, that is, they satisfy the following property: for all $C \in \mathrm{K}_{\mathrm{tac}}(R)$ and $D \in \mathrm{K}_{\mathrm{tac}}(Q)$ there exists a bijection

$$
\operatorname{Hom}_{Q}(D, T C) \rightarrow \operatorname{Hom}_{R}(S D, C)
$$

which is natural in each variable.

Definition 2.2.6. Let $R=Q /\left(f_{1}, \ldots, f_{c}\right)$ and let $C, D \in \mathrm{K}_{\mathrm{tac}}(R)$. Then the Rank Variety $W(Q, f, C, D)$ of a pair of complexes $C, D \in \mathrm{K}_{\mathrm{tac}}(R)$ is given by

$$
W(Q, f, C, D)=\left\{\bar{a} \in \mathbb{k}^{c} \mid \operatorname{Hom}_{Q_{a}}\left(T C, \Sigma^{i} T D\right)\right) \neq 0
$$

for infinitely many $i>0\} \cup\{0\}$ 
where $a=\left(a_{1}, \ldots, a_{c}\right) \in Q^{c}$ denotes some lifting of $\bar{a}=\left(\overline{a_{1}}, \ldots, \overline{a_{c}}\right) \in \mathbb{k}^{c}$ and $Q_{a}=$ $Q / f_{a} Q$ with $f_{a}=a_{1} f_{1}+\cdots+a_{c} f_{c} \in Q$.

While it is clear that $V(Q, f, C, D)$ is an algebraic variety, it is not at all obvious from the definition that $W(Q, f, C, D)$ is a closed set. Furthermore, some of the properties discussed in section 3.3 are proved using the rank variety definition while others are proved using the support variety definition. When we discuss realizability of support in chapter 4, we mainly focus on the cohomology operators in the support variety definition. However, we also need to know the support variety of a complete resolution of the residue field. This is very easily computed using the rank variety and a result of Eisenbud $[15,2]$. We will now spend the next chapter building the tools necessary to show that $V(Q, f, C, D)$ is the same as $W(Q, f, C, D)$. 


\section{Chapter 3}

\section{Equivalence of Support and Rank Varieties}

Our main goal in this chapter is to show that the support and rank varieties of a pair of totally acyclic complexes are equivalent; translating the Avrunin-Scott theorem to $\mathrm{K}_{\mathrm{tac}}(R)$. We use the pair of adjoint triangle functors $T: \mathrm{K}_{\mathrm{tac}}(R) \rightarrow \mathrm{K}_{\mathrm{tac}}(Q)$ and $S: \mathrm{K}_{\mathrm{tac}}(Q) \rightarrow \mathrm{K}_{\mathrm{tac}}(R)[10,2.1]$ to identify morphisms in $\mathrm{K}_{\mathrm{tac}}(R)$ with morphisms in $\mathrm{K}_{\mathrm{tac}}(Q)$. We then use this identification of morphisms along with an iterated lifted mapping cone to prove a key finite generation result relating $\operatorname{Hom}_{Q}$ to $\operatorname{Hom}_{R}$ for a pair of totally acyclic complexes. Finally, we take advantage of this finite generation result in a slightly modified version of the proof by Avramov and Buchweitz [2, 2.5] to prove the equivalence of varieties.

\subsection{Finite Generation}

The proof of the equivalence of support varieties of modules over a complete intersection ring requires a theorem that states for any pair of $R$-modules $M, N$, the graded $R$-module $\operatorname{Ext}_{Q}^{*}(M, N)$ is finitely generated if and only if the graded $R\left[\chi_{1}, \ldots, \chi_{c}\right]$-module $\operatorname{Ext}_{R}^{*}(M, N)$ is finitely generated, where $R\left[\chi_{1}, \ldots, \chi_{c}\right]$ is the ring of cohomology operators. The 'if' direction of this theorem was proved by Avramov, Gasharov, and Peeva [3, 4.2] and the 'only if' direction was proved by Gulliksen [17, 3.1] cf. [1, 2.1].

Our main goal in this section is to translate the Gulliksen and AvramovGasharov-Peeva finiteness theorem for Ext to the setting of totally acyclic chain

complexes using the triangulated structure of $\mathrm{K}_{\text {tac }}(R)$. The following generalization 
of a result by Avramov [1,2.3] gives a nice condition for showing the finiteness of a graded $A[x]$-module given the finiteness of a graded $A$-module.

Lemma 3.1.1. Let $A$ be a non-negatively graded noetherian ring and let $F$ be a graded $A$-module and $E$ be a graded $A[x]$-module. Regarding $F$ as a graded $A[x]$-module by the map $s: A[x] \rightarrow A$ where $s(x)=0$, graded in total degree $\left|a x^{n}\right|=|a|+n|x|$ for any homogeneous element $a \in A$. Let there exist graded $A[x]$-module homomorphisms $\psi: E \rightarrow F$ and $\phi: F \rightarrow E$ and an exact sequence of the form

$$
\stackrel{\psi}{\longrightarrow} F^{i} \stackrel{\phi}{\longrightarrow} E^{i+|\phi|} \stackrel{x}{\longrightarrow} E^{i+|\phi|+|x|} \stackrel{\psi}{\longrightarrow} F^{i+|\phi|+|x|+|\psi|} \stackrel{\phi}{\longrightarrow} .
$$

Then $F$ is a finitely generated graded A-module if and only if $E$ is a finitely generated graded $A[x]$-module.

Proof. First assume $F$ is finitely generated as an $A$-module. Since $F$ is noetherian, $\operatorname{Im}(\psi)$ is finitely generated. Let $\psi\left(e_{1}\right), \ldots, \psi\left(e_{m}\right)$ be its generators. Now consider $G=A e_{1}+\cdots+A e_{m}$ as an $A[x]$-graded submodule of $E$. Now let $e \in E$. Then $\psi(e)=a_{1} \psi\left(e_{1}\right)+\cdots+a_{m} \psi\left(e_{m}\right)$. Thus $e-\left(a_{1} e_{1}+\cdots+a_{n} e_{n}\right) \in \operatorname{Ker}(\psi)$, so $E \subseteq$ $G+\operatorname{Ker}(\psi)$. The opposite containment is obvious, thus $E=G+\operatorname{Ker}(\psi)$. But $\operatorname{Ker}(\psi)=\operatorname{Im}(x)=x E$. Thus $E=G+x E$. Iterating yields

$$
E=\sum_{i=0}^{n} x^{i} G+x^{n+1} E .
$$

For any homogeneous element $e \in E$ and for $n \gg 0, e \notin x^{n+1} E$ so we get $E=$ $(A[x] G)$. Thus $E$ is a finitely generated $A[x]$-module.

Now conversely assume that $E$ is a finitely generated $A[x]$-module. Consider the short exact sequence of graded $A[x]$-modules,

$$
0 \rightarrow E / \operatorname{Ker}(\psi) \rightarrow F \rightarrow \operatorname{Im}(\phi) \rightarrow 0
$$

Since $E$ is noetherian, both $E / \operatorname{Ker}(\psi)$ and $\operatorname{Im}(\phi)$ are finitely generated, so $F$ is finitely generated as an $A[x]$ module. So for any $f \in F, f=p_{1} f_{1}+\cdots+p_{n} f_{n}$ where 
$p_{i} \in A[x]$. But $x \in \operatorname{Ann}(F)$, so $p_{i} f_{i}=a_{i} f_{i}$ where $a_{i} \in A$ is the constant term of $p_{i}$. Thus $F$ is finitely generated as an $A$ module.

Remark 3.1.2. Since $A$ is assumed to be non-negatively graded and $|x|>0, F^{i}, E^{i}=0$ for $i<0$.

We now need to construct such a long exact sequence of Hom modules. To prove Gulliken's original theorem, Avramov [1, 2.1] uses a complicated homotopy construction by Shamash and Eisenbud. However, we can instead use the triangulated structure of $\mathrm{K}_{\mathrm{tac}}(R)$ to create the long exact sequence. First, we will need a few tools for handling the triangulated structure.

\section{The Lifted Mapping Cone}

Given a chain complex $C$ over the ring $R=Q /\left(f_{1}, \ldots, f_{c}\right)$, we cannot expect that a lifting of $C$ to $Q$ will itself be a complex. However, we can find liftings of mapping cones of the $t_{i}^{\prime} s$ that are complexes.

Lemma 3.1.3. Let $Q$ be a local ring, $R=Q /(f)$ where $f$ is a nonzerodivisor. Also let $C \in \mathrm{K}_{\mathrm{tac}}(R)$ with $\tilde{d}_{n-1}^{C} \circ \tilde{d}_{n}^{C}=\tilde{t}_{n} f$ so that $t=\sigma_{C}^{2}\left(\tilde{t} \otimes_{Q} R\right)$ is a chain map from $C$ to $\Sigma^{2} C$. Then there exists a lifting, $\widetilde{\operatorname{Cone}(t)}$, to $Q$ of Cone $(t)$ such that the lifting is a complex. Furthermore this complex is exact.

Remark 3.1.4. For simplicity of notation we will refer to $\widetilde{C o n e}(t)$ as $C^{\sharp}$.

Proof. Consider the mapping cone differential:

$$
d_{n}^{\text {Cone }(t)}=\left(\begin{array}{cc}
d_{n}^{\Sigma C} & 0 \\
t_{n-1}\left(\sigma_{C}^{1}\right)_{n-1}^{-1} & d_{n}^{\Sigma^{2} C}
\end{array}\right) .
$$


Then lift the mapping cone to $Q$ with the differential:

$$
d_{n}^{C^{\sharp}}=\left(\begin{array}{cc}
\tilde{d}_{n}^{\Sigma C} & -f\left(\tilde{\sigma}_{C}^{1}\right)_{n-2}\left(\tilde{\sigma}_{C}^{2}\right)_{n-2}^{-1} \\
\left(\tilde{\sigma}_{C}^{2}\right)_{n-3} \tilde{t}_{n-1}\left(\tilde{\sigma}_{C}^{1}\right)_{n-1}^{-1} & \tilde{d}_{n}^{\Sigma^{2} C}
\end{array}\right)
$$

where $\tilde{\sigma} \otimes_{Q} R=\sigma$. To see that this lifting is a $Q$-complex, we need to check that the composition of two consecutive differentials is zero.

$$
\begin{gathered}
d_{n-1}^{C^{\sharp}} \circ d_{n}^{C^{\sharp}}=\left(\begin{array}{cc}
a & b \\
c & d
\end{array}\right) \\
\text { where } \\
a=\tilde{d}_{n-1}^{\Sigma C} \tilde{d}_{n}^{\Sigma C}-f\left(\tilde{\sigma}_{C}^{1}\right)_{n-3} \tilde{t}_{n-1}\left(\tilde{\sigma}_{C}^{1}\right)_{n-1}^{-1} \\
b=-\tilde{d}_{n-1}^{\Sigma C}\left(\tilde{\sigma}_{C}^{1}\right)_{n-1}\left(\tilde{\sigma}_{C}^{2}\right)_{n-1}^{-1} f-f\left(\tilde{\sigma}_{C}^{1}\right)_{n-3}\left(\tilde{\sigma}_{C}^{2}\right)_{n-3}^{-1} \tilde{d}_{n}^{\Sigma^{2} C} \\
c=\left(\tilde{\sigma}_{C}^{2}\right)_{n-4} \tilde{t}_{n-2}\left(\tilde{\sigma}_{C}^{1}\right)_{n-2}^{-1} \tilde{d}_{n}^{\Sigma C}+\tilde{d}_{n-2}^{\Sigma^{2} C}\left(\tilde{\sigma}_{C}^{2}\right)_{n-3} \tilde{t}_{n-1}\left(\tilde{\sigma}_{C}^{1}\right)_{n-1}^{-1} \\
d=-f\left(\tilde{\sigma}_{C}^{2}\right)_{n-4} \tilde{t}_{n-2}\left(\tilde{\sigma}_{C}^{2}\right)_{n-2}^{-1}+\tilde{d}_{n-1}^{\Sigma^{2} C} \tilde{d}_{n}^{\Sigma^{2} C} .
\end{gathered}
$$

We also know that

$$
\tilde{d}_{n-1}^{\Sigma C} \circ \tilde{d}_{n}^{\Sigma C}=f\left(\tilde{\sigma}_{C}^{1}\right)_{n-3} \tilde{t}_{n-1}\left(\tilde{\sigma}_{C}^{1}\right)_{n-1}^{-1} \text { and } \tilde{d}_{n-1}^{\Sigma^{2} C} \circ \tilde{d}_{n}^{\Sigma^{2} C}=f\left(\tilde{\sigma}_{C}^{2}\right)_{n-4} \tilde{t}_{n-2}\left(\tilde{\sigma}_{C}^{2}\right)_{n-2}^{-1}
$$

therefore $a$ and $d$ are zero. Furthermore, since $\tilde{d}_{n-1}^{\Sigma C}=-\left(\tilde{\sigma}_{C}^{1}\right)_{n-3} \tilde{d}_{n-2}^{C}\left(\tilde{\sigma}_{C}^{1}\right)_{n-1}^{-1}$ and $\tilde{d}_{n}^{\Sigma^{2} C}=\left(\tilde{\sigma}_{C}^{2}\right)_{n-3} \tilde{d}_{n-2}^{C}\left(\tilde{\sigma}_{C}^{2}\right)_{n}^{-1}$, we have

$$
\begin{aligned}
b & =-\tilde{d}_{n-1}^{\Sigma C}\left(\tilde{\sigma}_{C}^{1}\right)_{n-1}\left(\tilde{\sigma}_{C}^{2}\right)_{n-1}^{-1} f-f\left(\tilde{\sigma}_{C}^{1}\right)_{n-3}\left(\tilde{\sigma}_{C}^{2}\right)_{n-3}^{-1} \tilde{d}_{n}^{\Sigma^{2} C} \\
& =-\left(\tilde{\sigma}_{C}^{1}\right)_{n-3} \tilde{d}_{n-2}^{C}\left(\tilde{\sigma}_{C}^{2}\right)_{n}^{-1}+\left(\tilde{\sigma}_{C}^{1}\right)_{n-3} \tilde{d}_{n-2}^{C}\left(\tilde{\sigma}_{C}^{2}\right)_{n}^{-1}=0
\end{aligned}
$$


If we multiply $c$ by $f$ we get,

$$
\begin{aligned}
& f c=f\left(\left(\tilde{\sigma}_{C}^{2}\right)_{n-4} \tilde{t}_{n-2}\left(\tilde{\sigma}_{C}^{1}\right)_{n-2}^{-1} \tilde{d}_{n}^{\Sigma C}+\tilde{d}_{n-1}^{\Sigma^{2} C}\left(\tilde{\sigma}_{C}^{2}\right)_{n-3} \tilde{t}_{n-1}\left(\tilde{\sigma}_{C}^{1}\right)_{n-1}^{-1}\right)= \\
& -\left(\tilde{\sigma}_{C}^{2}\right)_{n-4} \tilde{d}_{n-2}^{C} \tilde{d}_{n-1}^{C} \tilde{d}_{n}^{C}\left(\tilde{\sigma}_{C}^{1}\right)_{n-1}^{-1}+\left(\tilde{\sigma}_{C}^{2}\right)_{n-4} \tilde{d}_{n-2}^{C} \tilde{d}_{n-1}^{C} \tilde{d}_{n}^{C}\left(\tilde{\sigma}_{C}^{1}\right)_{n-1}^{-1}=0 .
\end{aligned}
$$

But $f$ is a non-zerodivisor, thus $c$ is also zero. Thus $d_{n-1}^{C^{\sharp}} \circ d_{n}^{C^{\sharp}}=0$ and hence the lifting of the cone is a complex.

Now to see that the complex is exact, let $\tilde{x} \in \operatorname{Ker} d_{n}^{C^{\sharp}}$. Then $x=\tilde{x}+f \operatorname{Cone}(t) \in$ $\operatorname{Ker} d_{n}^{\operatorname{Cone}(t)}$. We know that Cone $(t)$ is exact, thus there exists $y \in \operatorname{Cone}(t)_{n+1}$ such that $d_{n}^{\text {Cone }(t)}(y)=x$. Choose $\tilde{y} \in C_{n+1}^{\sharp}$ such that $y=\tilde{y}+f \operatorname{Cone}(t)_{n+1}$. Therefore $d_{n+1}^{C^{\sharp}}(\tilde{y})-\tilde{x} \in f C_{n}^{\sharp}$. So there exists $\tilde{z} \in C_{n}^{\sharp}$ such that $d_{n+1}^{C^{\sharp}}(\tilde{y})-\tilde{x}=f \tilde{z}$. Applying $d_{n}^{C^{\sharp}}$ to both sides we get

$$
f d_{n}^{C^{\sharp}}(\tilde{z})=d_{n}^{C^{\sharp}}(f \tilde{z})=d_{n}^{C^{\sharp}}\left(d_{n+1}^{C^{\sharp}}(\tilde{y})-\tilde{x}\right)=0 .
$$

Since $f$ is a non-zerodivizor, we can conclude that $d_{n}^{C^{\sharp}}(\tilde{z})=0$, so $\tilde{z} \in \operatorname{Ker} d_{n}^{C^{\sharp}}$. This implies that

$$
\operatorname{Ker} d_{n}^{C^{1}} \subseteq \operatorname{Im} d_{n+1}^{C^{\sharp}}+f \operatorname{Ker} d_{n}^{C^{\sharp}} .
$$

We already know that $\operatorname{Im} d_{n+1}^{C^{\sharp}} \subseteq \operatorname{Ker} d_{n}^{C^{\sharp}}$, thus by Nakayama's lemma, $\operatorname{Ker} d_{n}^{C^{\sharp}}=$ $\operatorname{Im} d_{n+1}^{C^{\sharp}}$.

The above lemma gives a lifting when $R=Q /(f)$. However, we would like to be able to deal with the case where $R=Q /\left(f_{1}, \ldots, f_{c}\right)$. We can apply an induction argument to get a complex over $Q$ in this case. In particular, we can take $R_{k}=$ $Q /\left(f_{k}, \ldots, f_{c}\right)$ so that $R_{k}=R_{k-1} /\left(f_{k}\right)$ and so $R=R_{1}=R_{2} /\left(f_{1}\right)$. Now let $C \in$ $\mathrm{K}_{\text {tac }}(R)$ and lift $C$ to $R_{2}$ with $\left(\tilde{d}^{C}\right)^{2}=\tilde{u}_{1} f_{1}$. We know that the complex Cone $\left(u_{1}\right) \in$ $\mathrm{K}_{\text {tac }}(R)$ lifts to a complex $C^{\sharp} \in \mathrm{K}_{\text {tac }}\left(R_{2}\right)$. Now we can lift this complex to get

$$
\left(\tilde{d}^{C^{\sharp}}\right)^{2}=\tilde{u}_{2} f_{2} \text { where } u_{2}=\sigma_{C^{\sharp}}^{2}\left(\widetilde{u}_{2} \otimes_{Q} R\right) \text { and } u_{2}: C^{\sharp} \rightarrow \Sigma^{2} C^{\sharp} \text {. }
$$


But now we can consider $\operatorname{Cone}\left(u_{2}\right) \in \mathrm{K}_{\text {tac }}\left(R_{2}\right)$ and lift it to $C^{\sharp 2} \in \mathrm{K}_{\text {tac }}\left(R_{3}\right)$. We can continue this process until we reach $C^{\sharp c} \in \mathrm{K}_{\text {tac }}(Q)$ with maps given by

$$
d_{n}^{C^{\sharp c}}=\left(\begin{array}{cc}
\tilde{d}_{n}^{\Sigma C^{\sharp c-1}} & -f_{c}\left(\widetilde{\sigma}_{C^{\sharp c-1}}^{1}\right)_{n-2}\left(\widetilde{\sigma}_{C^{\sharp c-1}}^{2}\right)_{n-2}^{-1} \\
\left(\widetilde{\sigma}_{C^{\sharp c-1}}^{2}\right)_{n-3}\left(\widetilde{u}_{c}\right)_{n}\left(\widetilde{\sigma}_{C^{\sharp c-1}}^{1}\right)_{n-1}^{-1} & \tilde{d}_{n}^{\Sigma^{2} C^{\sharp c-1}}
\end{array}\right) .
$$

The notation for this iterated lifted cone is somewhat unwieldy. The next lemma will allow us to replace the iterated cone by $T C$.

Lemma 3.1.5. Let $R=Q /\left(f_{1}, \ldots, f_{c}\right)$ and let $C \in \mathrm{K}_{\mathrm{tac}}(R)$. Also let $C^{\sharp c}$ be defined as above. Then $C^{\sharp c} \simeq T C$.

Proof. First consider the case where $R=Q /\left(f_{1}\right)$ and consider the following $Q$ complex

$$
\cdots \stackrel{d_{2}^{C^{\sharp}}}{\longrightarrow} C_{1}^{\sharp} \stackrel{\left(\tilde{d}_{1}^{C}\left(\widetilde{\sigma}_{C}^{1}\right)_{1}^{-1} f\left(\widetilde{\sigma}_{C}^{2}\right)_{0}^{-1}\right)}{\longrightarrow} \widetilde{C}_{0} \stackrel{d_{0}^{C} \circ \pi}{\longrightarrow} \operatorname{Im} d_{0}^{C} \longrightarrow 0 .
$$

Where $d_{n}^{C^{\sharp}}$ is as defined above and $\pi$ is the natural surjection onto $C_{0}$. This complex agrees with $C^{\sharp}$ to the left. The complex is clearly exact to the left of $C_{1}^{\sharp}$. To show it is exact at $C_{1}^{\sharp}$, let $\left(x_{1}, x_{2}\right) \in \operatorname{Ker}\left(\tilde{d}_{1}^{C}\left(\tilde{\sigma}_{C}^{1}\right)_{1}^{-1} f\left(\tilde{\sigma}_{C}^{2}\right)_{0}^{-1}\right)$. Then

$$
\tilde{d}_{1}^{C}\left(\tilde{\sigma}_{C}^{1}\right)_{1}^{-1}\left(x_{1}\right)+f\left(\tilde{\sigma}_{C}^{2}\right)_{0}^{-1}\left(x_{2}\right)=0
$$

which implies $d_{1}^{C}\left(\sigma_{C}^{1}\right)_{1}^{-1}\left(x_{1}\right)=0$. But $C$ is exact so $\left(\sigma_{C}^{1}\right)_{1}^{-1}\left(x_{1}\right) \in \operatorname{Im} d_{2}^{C}$ and so there exists $y_{1} \in(\Sigma C)_{2}$ such that

$$
d_{2}^{C}\left(\sigma_{C}^{1}\right)_{1}^{-1}\left(y_{1}\right)-\left(\sigma_{C}^{1}\right)_{1}^{-1}\left(x_{1}\right)=0
$$

Now lifting to $Q$ there exists $y_{2} \in\left(\Sigma^{2} C\right)_{2}$ such that

$$
\tilde{d}_{2}^{C}\left(\tilde{\sigma}_{C}^{1}\right)_{1}^{-1}\left(y_{1}\right)-\left(\tilde{\sigma}_{C}^{1}\right)_{1}^{-1}\left(x_{1}\right)=f\left(\tilde{\sigma}_{C}^{2}\right)_{0}^{-1}\left(y_{2}\right)
$$

Rearranging this and applying $\left(\tilde{\sigma}_{C}^{2}\right)_{2} \tilde{d}_{1}^{C}$ to both sides we get

$$
f\left(\tilde{\sigma}_{C}^{2}\right)_{2} \tilde{t}_{1}\left(\tilde{\sigma}_{C}^{1}\right)_{2}^{-1}\left(y_{1}\right)+f\left(\tilde{\sigma}_{C}^{2}\right)_{2} \tilde{d}_{1}^{C}\left(\tilde{\sigma}_{C}^{1}\right)_{1}^{-1}\left(y_{2}\right)=\left(\tilde{\sigma}_{C}^{2}\right)_{2} \tilde{d}_{1}^{C}\left(\tilde{\sigma}_{C}^{1}\right)_{1}^{-1}\left(x_{1}\right)
$$


But we know that $\tilde{d}_{1}^{C}\left(\tilde{\sigma}_{C}^{1}\right)_{1}^{-1}\left(x_{1}\right)=-f\left(\tilde{\sigma}_{C}^{2}\right)_{0}^{-1}\left(x_{2}\right)$ so we have

$$
\left(\tilde{\sigma}_{C}^{2}\right)_{0} \tilde{t}_{1}\left(\tilde{\sigma}_{C}^{1}\right)_{1}^{-1}\left(y_{1}\right)+\widetilde{d_{1}^{C}}\left(\tilde{\sigma}_{C}^{1}\right)_{1}^{-1}\left(y_{2}\right)=x_{2}
$$

Therefore $\left(x_{1}, x_{2}\right) \in \operatorname{Im} d_{2}^{C^{\sharp}}$.

To see that the complex is exact at $\widetilde{C_{0}}$, let $\tilde{x} \in \operatorname{Ker} d_{0}^{C} \circ \pi$. Then for $\pi(\tilde{x})=$ $\tilde{x}+f \widetilde{C_{0}}, x \in \operatorname{Ker} d_{0}^{C}$. Since $C$ is an exact complex, there exists $y \in C_{1}$ such that $d_{1}^{C}(y)=x$. Now choose $\tilde{y} \in \widetilde{C_{1}}$ such that $y=\tilde{y}+f \widetilde{C_{1}}$. Then $\tilde{d}_{1}^{C}(\tilde{y})-\tilde{x} \in f \widetilde{C_{1}}$. So there exists $a \in \widetilde{C}_{1}$ such that $\tilde{d}_{1}^{C}(\tilde{y})-\tilde{x}=a f$. Therefore

$$
\tilde{d}_{1}^{C}\left(\tilde{\sigma}_{C}^{1}\right)_{1}^{-1}\left(\left(\tilde{\sigma}_{C}^{1}\right)_{1}(\tilde{y})\right)-f\left(\tilde{\sigma}_{C}^{2}\right)_{0}^{-1}\left(\left(\tilde{\sigma}_{C}^{2}\right)_{0}(a)\right)=\tilde{x}
$$

which shows that $\tilde{x} \in \operatorname{Im}\left(\tilde{d}_{1}^{C}\left(\tilde{\sigma}_{C}^{1}\right)_{1}^{-1} f\left(\tilde{\sigma}_{C}^{2}\right)_{0}^{-1}\right)$. Thus the complex is exact and hence is a complete resolution of $\operatorname{Im} d_{0}^{C}$. So by definition, $C^{\sharp} \simeq T C$.

Notice if we let $R=Q /\left(f_{1}, \ldots, f_{c}\right)$ and consider the functors

$$
T_{i}: \mathrm{K}_{\mathrm{tac}}\left(Q /\left(f_{i}, \ldots, f_{c}\right)\right) \rightarrow \mathrm{K}_{\mathrm{tac}}\left(Q /\left(f_{i+1}, \ldots, f_{c}\right)\right)
$$

then $T=T_{1} \circ \ldots \circ T_{c}$ since $\operatorname{Im} d_{0}^{C} \cong \operatorname{Im} d_{0}^{T_{i} C}$. Thus in general, $C^{\sharp c} \simeq T C$.

\section{The Long Exact Sequence}

We now have the tools to prove the main result of this section.

Theorem 3.1.6. Let $R=Q /\left(f_{1}, \ldots, f_{c}\right)$ where $Q$ is a local noetherian ring, $f_{1}, \ldots, f_{c}$ is a $Q$-regular sequence, and let $C, D \in \mathrm{K}_{\mathrm{tac}}(R)$. Then $\bigoplus_{i \geq 0} \operatorname{Hom}_{Q}\left(T C, \Sigma^{i} T D\right)$ is finitely generated as an $R$-module if and only if $\bigoplus_{i \geq 0} \operatorname{Hom}_{R}\left(C, \Sigma^{i} D\right)$ is finitely generated as an $R\left[\chi_{1}, \ldots, \chi_{c}\right]$-module

Proof. First consider the case where $R=Q /\left(f_{1}\right)$ where $f_{1}$ is a single nonzerodivisor. Consider the exact triangle

$$
C \rightarrow \Sigma^{2} C \rightarrow \text { Cone }\left(t_{1}\right) \rightarrow \Sigma C \text {. }
$$


Since $\mathrm{K}_{\mathrm{tac}}(R)$ is a triangulated category, we can apply the contravariant Hom functor to the exact triangle to get the long exact sequence

$$
\begin{gathered}
\ldots \rightarrow \operatorname{Hom}_{R}\left(\Sigma^{i} C, D\right) \rightarrow \operatorname{Hom}_{R}\left(\Sigma^{i-1} \operatorname{Cone}\left(t_{1}\right), D\right) \rightarrow \\
\operatorname{Hom}_{R}\left(\Sigma^{i+1} C, D\right) \rightarrow \operatorname{Hom}_{R}\left(\Sigma^{i-1} C, D\right) \rightarrow \ldots
\end{gathered}
$$

for any $D \in \mathrm{K}_{\mathrm{tac}}(R)$. By Lemma 3.1.3

$$
\Sigma^{i} \operatorname{Cone}\left(t_{1}\right) \cong S \Sigma^{i} C^{\sharp} \in \mathrm{K}_{\mathrm{tac}}(R) .
$$

Also, by Theorem 2.2.5

$$
\operatorname{Hom}_{R}\left(\Sigma^{i} S C^{\sharp}, D\right) \cong \operatorname{Hom}_{Q}\left(\Sigma^{i} C^{\sharp}, T D\right) .
$$

So we now have the long exact sequence

$$
\begin{aligned}
\ldots \rightarrow & \operatorname{Hom}_{R}\left(\Sigma^{i} C, D\right) \rightarrow \operatorname{Hom}_{Q}\left(\Sigma^{i-1} C^{\sharp}, T D\right) \rightarrow \\
& \operatorname{Hom}_{R}\left(\Sigma^{i+1} C, D\right) \rightarrow \operatorname{Hom}_{R}\left(\Sigma^{i-1} C, D\right) \rightarrow \ldots .
\end{aligned}
$$

So lastly we can apply Lemma 3.1.1 and find that $\bigoplus_{i \geq 0} \operatorname{Hom}_{Q}\left(\Sigma^{-i} C^{\sharp}, T D\right)$ is finitely generated as an $R$-module if and only if $\bigoplus_{i \geq 0} \operatorname{Hom}_{R}\left(\Sigma^{-i} C, D\right)$ is finitely generated as an $R[\chi]$-module.

Now let the statement hold for $R^{\prime}=Q^{\prime} /\left(f_{1}, \ldots, f_{k-1}\right)$. Let $R^{\prime}=Q$ and $R=$ $Q /\left(f_{k}\right)$. We can now apply the same argument as above to the exact triangle

$$
C^{\sharp k-1} \rightarrow \Sigma^{2} C^{\sharp k-1} \rightarrow \operatorname{Cone}\left(u_{k}\right) \rightarrow \Sigma C^{\sharp k-1}
$$

where $u_{k}$ is the Eisenbud operator of $C^{\sharp k-1}$ for $k>1$ and $u_{1}=t_{1}$. This yields that $\bigoplus_{i \geq 0} \operatorname{Hom}_{Q^{\prime}}\left(\Sigma^{-i} C^{\sharp k}, T D\right)$ is finitely generated as an $R$-module if and only if $\bigoplus_{i \geq 0} \operatorname{Hom}_{R}\left(\Sigma^{-i} C, D\right)$ is finitely generated as an $R\left[\chi_{1}, \ldots, \chi_{k}\right]$-module. This is equivalent to the statement $\bigoplus_{i \geq 0} \operatorname{Hom}_{Q^{\prime}}\left(C^{\sharp k}, \Sigma^{i} T D\right)$ is finitely generated as an $R$-module if and only if $\bigoplus_{i \geq 0} \operatorname{Hom}_{R}\left(C, \Sigma^{i} D\right)$ is finitely generated as an $R\left[\chi_{1}, \ldots, \chi_{k}\right]$-module. We can now use Lemma 3.1.5 to replace $C^{\sharp k}$ by $T C$ and so the theorem holds. 


\subsection{Avrunin-Scott for $\mathrm{K}_{\mathrm{tac}}(R)$}

We are now ready to show that the support variety and rank variety of a pair of totally acyclic complexes are the same, translating the Avrunin-Scott theorem $[6$, 1.1] to the setting of $\mathrm{K}_{\mathrm{tac}}(R)$. The proof follows the same logic that Avramov and Buchweitz $[2,2.5]$ use to prove a similar equivalence for the support variety of a pair of modules over a complete intersection ring.

Theorem 3.2.1. Let $R=Q /\left(f_{1}, \ldots, f_{c}\right)$ where $(Q, m, \mathbb{k})$ is a regular local ring and $f_{1}, \ldots, f_{c}$ is a regular sequence. Then $V(Q, f, C, D)=W(Q, f, C, D)$.

Proof. Since $0 \in V(Q, f, C, D)$, we can assume that $a_{i} \neq 0$ for some $i$. Since $f$ is a regular sequence, there exists a regular sequence $f^{\prime}=f_{1}^{\prime}, \ldots, f_{c}^{\prime}$ such that $f$ and $f^{\prime}$ generate the same ideal and $\left(a_{1}, \ldots, a_{n}\right)=(0, \ldots, 0,1)$. This means that $f_{a}=f_{c}^{\prime}$, so we only have to show that $\mathbb{k}(0, \ldots, 0,1) \subseteq V(Q, f, C, D)$ if and only if $\operatorname{Hom}_{Q_{a}}\left(T C, \Sigma^{i} T D\right) \neq 0$ for infinitely many $i$. We will actually prove the contrapositive of the statement. That is, $\operatorname{Hom}_{Q_{a}}\left(T C, \Sigma^{i} T D\right)=0$ for $i \gg 0$ if and only if $\mathbb{k}(0, \ldots, 0,1) \nsubseteq V(Q, f, C, D)$.

Let $Q^{\prime}=Q /\left(f_{c}^{\prime}\right)$ and recall the notation from definition 2.2.1, $S=R\left[\chi_{1}, \ldots, \chi_{c}\right]$ and $E=\bigoplus_{i \geq 0} \operatorname{Hom}_{R}\left(C, \Sigma^{i} D\right) \otimes_{R} \mathbb{k}$. The sequence $f_{1}^{\prime}, \ldots, f_{c-1}^{\prime}$ is regular on $Q^{\prime}$ so we can consider the cohomology ring of $S^{\prime}=R\left[\chi_{1}^{\prime}, \ldots, \chi_{c-1}^{\prime}\right]$ of $Q^{\prime}$ defined by this sequence. By Proposition 2.1.6, the action of $\chi_{k}^{\prime}$ on $E$ agrees with the action of $\chi_{k}$ and thus by mapping $\chi_{k}^{\prime}$ to $\chi_{k}$ we can consider $S^{\prime}$ to be a sub ring of $S$.

By Theorem 3.1.6, we know that $\operatorname{Hom}_{Q^{\prime}}\left(T C, \Sigma^{i} T D\right)=0$ for $i \gg 0$ if and only if the $S^{\prime}$-module $\bigoplus_{i \geq 0} \operatorname{Hom}_{R}\left(C, \Sigma^{i} D\right)$ is finitely generated. But by Nakayama's Lemma, this happens if and only if $E$ is finitely generated over $S^{\prime} \otimes_{R}$ k. This is equivalent to $E^{\prime}=E / E\left(\chi_{1}^{\prime}, \ldots, \chi_{c-1}^{\prime}\right) \cong E / E\left(\chi_{1}, \ldots, \chi_{c-1}\right)$ being finitely generated over $\left(S^{\prime} \otimes_{R} \mathbb{k}\right) /\left(\chi_{1}^{\prime}, \ldots, \chi_{c-1}^{\prime}\right) \cong \mathbb{k}$. 
Now let $\mathcal{R}=S \otimes_{R} \mathbb{k}$ and $\mathcal{R}^{\prime}=\mathcal{R} /\left(\chi_{1}, \ldots, \chi_{c-1}\right)$. Then $\operatorname{rank}_{\mathbb{k}} E^{\prime}$ is finite if and only if $\operatorname{Supp}_{\mathcal{R}}\left(E^{\prime}\right)=\left\{\left(\chi_{1}, \ldots, \chi_{c}\right)\right\}$. Also since $\mathrm{E}$ is finitely generated over $\mathcal{R}$ and $E^{\prime}=E \otimes_{R} \mathcal{R}$, we have

$$
\operatorname{Supp}_{\mathcal{R}}\left(E^{\prime}\right)=\operatorname{Supp}_{\mathcal{R}}\left(E \otimes_{R} \mathcal{R}^{\prime}\right)=\operatorname{Supp}_{\mathcal{R}}(E) \bigcap \operatorname{Supp}_{\mathcal{R}}\left(\mathcal{R}^{\prime}\right) .
$$

This is true if and only if

$$
\sqrt{\operatorname{Ann} E} \bigcap \sqrt{\operatorname{Ann} \mathcal{R}^{\prime}}=\left(\chi_{1}, \ldots, \chi_{c}\right) .
$$

Since $Z\left(\operatorname{Ann}_{\mathcal{R}} E\right)=V(Q, f, C, D), Z\left(\operatorname{Ann}_{\mathcal{R}} \mathcal{R}^{\prime}\right)=\mathbb{k}(0, \ldots, 0,1)$, and $Z\left(\operatorname{Ann}_{\mathcal{R}}\left(E^{\prime}\right)\right)=$ 0 , the Nullstellensatz implies the previous line is true if and only if

$$
V(Q, f, C, D) \bigcap \mathbb{k}(0, \ldots, 0,1)=\{0\} \in \mathbb{k}^{c} .
$$

So looking at the above list of if and only if statements, we now have $\operatorname{Hom}_{Q^{\prime}}\left(T C, \Sigma^{i} T D\right)=0$ for $i \gg 0$ if and only if $\mathbb{k}(0, \ldots, 0,1) \nsubseteq V(Q, f, C, D)$.

\subsection{Properties of Support/Rank Varieties}

We will now prove a few basic results about support/rank varieties in $\mathrm{K}_{\mathrm{tac}}(R)$. The same properties hold for support varieties of modules over a complete intersection ring and the proofs are similar $[2,5.6]$ cf. $[9,2.2]$.

The first theorem provides a nice analogue to Dade's Lemma [14] for $\mathrm{K}_{\mathrm{tac}}(R)$. Theorem 3.3.1. Let $R=Q /\left(f_{1}, \ldots, f_{c}\right)$ where $(Q, m, \mathbb{k})$ is a regular local ring and $f_{1}, \ldots, f_{c}$ is a $Q$-regular sequence. Also let $C, D \in \mathrm{K}_{\mathrm{tac}}(R)$. Then $\operatorname{Hom}_{R}\left(C, \Sigma^{i} D\right)=0$ for $i \gg 0$ if and only if $V(Q, f, C, D)=\{0\}$

Proof. Consider the long exact sequence of Hom from the proof of Theorem 3.1.6. If $\operatorname{Hom}_{R}\left(C, \Sigma^{n} D\right)=0$ for $n \gg 0$ then $\operatorname{Hom}_{Q_{a}}\left(T C, \Sigma^{n} T D\right)$ must also vanish for large $n$. Thus for any $\bar{a} \in \mathbb{k}^{c}$ such that $\bar{a} \neq 0, \bar{a} \notin V(Q, f, C, D)$. 
Now conversely assume $V(Q, f, C, D)=\{0\}$ and let $E=\bigoplus_{i \geq 0} \operatorname{Hom}_{R}\left(C, \Sigma^{i} D\right) \otimes_{R}$ k. Then $\sqrt{\operatorname{Ann} E}=m$ and so $m^{r} \subseteq$ Ann $E$ for some $r>0$. Therefore $m^{r} E=0$ and so for $n \gg 0$ we get $E_{n} \subseteq m^{r} E_{\leq n-r}=0$. Hence for $n \gg 0, E_{n}=\operatorname{Hom}_{R}\left(C, \Sigma^{n} D\right)=$ 0 .

Theorem 3.3.2. Let $R=Q /\left(f_{1}, \ldots, f_{c}\right)$ where $(Q, m, \mathbb{k})$ is a regular local ring and $f_{1}, \ldots, f_{c}$ is a $Q$-regular sequence. Also let $C, D \in \mathrm{K}_{\mathrm{tac}}(R)$. Then the following hold.

1. If $D$ is a complete resolution of the residue field $\mathbb{k}$, then

$$
V(Q, f, D, D)=\mathbb{k}^{c}
$$

2. $V(Q, f, C, D)=V(Q, f, C, C) \cap V(Q, f, D, D)$

3. If $D$ is a complete resolution of the field $\mathbb{k}$, then

$$
V(Q, f, C, D)=V(Q, f, C, C)
$$

4. If $C=\bigoplus_{p=1}^{r} C_{p}$ and $D=\bigoplus_{q=1}^{s} D_{q}$, then $V(Q, f, C, D)=\cup_{p, q} V\left(Q, f, C_{p}, D_{q}\right)$.

5. If $C \rightarrow C^{\prime} \rightarrow \operatorname{Cone}(\alpha) \rightarrow \Sigma C$ and $D \rightarrow D^{\prime} \rightarrow \operatorname{Cone}(\beta) \rightarrow \Sigma D$ are exact triangles in $\mathrm{K}_{\mathrm{tac}}(R)$, then we have the following inclusions.

$$
\begin{aligned}
V(Q, f, C, D) & \subseteq V\left(Q, f, C^{\prime}, D\right) \cup V(Q, f, \text { Cone }(\alpha), D) \\
V\left(Q, f, C^{\prime}, D\right) & \subseteq V(Q, f, C, D) \cup V(Q, f, \text { Cone }(\alpha), D) \\
V(Q, f, \operatorname{Cone}(\alpha), D) & \subseteq V(Q, f, C, D) \cup V\left(Q, f, C^{\prime}, D\right) \\
V(Q, f, C, D) & \subseteq V\left(Q, f, C, D^{\prime}\right) \cup V(Q, f, C, \text { Cone }(\beta)) \\
V\left(Q, f, C, D^{\prime}\right) & \subseteq V(Q, f, C, D) \cup V(Q, f, C, \text { Cone }(\beta)) \\
V(Q, f, C, \operatorname{Cone}(\beta)) & \subseteq V(Q, f, C, D) \cup V\left(Q, f, C, D^{\prime}\right)
\end{aligned}
$$

Proof. 
1. By $[15,2]$, proj $\operatorname{dim}_{Q_{a}} \mathbb{k}=\infty$ thus $\operatorname{Hom}_{Q_{a}}\left(T C, \Sigma^{n} T C\right) \neq 0$ for infinitely many $n$. Thus $V(Q, f, C, C)=\mathbb{k}^{c}$.

2. First let $\bar{a} \in V(Q, f, C, C) \cap V(Q, f, D, D)$ but $\bar{a} \notin V(Q, f, C, D)$. Then by Theorem 3.2.1, we have $\operatorname{Hom}_{Q_{a}}\left(T C, \Sigma^{n} T C\right) \neq 0$ and $\operatorname{Hom}_{Q_{a}}\left(T D, \Sigma^{n} T D\right) \neq 0$ for infinitely many $n$ while $\operatorname{Hom}_{Q_{a}}\left(T C, \Sigma^{n} T D\right)=0$ for $n \gg 0$. Thus $T C \simeq$ $0 \simeq T D$ but $\operatorname{Hom}_{Q_{a}}\left(T C, \Sigma^{n} T D\right)=0$ for $n \gg 0$, a contradiction. Therefore

$$
V(Q, f, C, C) \cap V(Q, f, D, D) \subseteq V(Q, f, C, D) .
$$

Now let $p(\chi) \in$ Ann $\bigoplus_{i \geq 0} \operatorname{Hom}_{R}\left(C, \Sigma^{i} C\right)$. Then we have $p\left(\operatorname{Hom}_{R}(t, D)\right)(\alpha) \sim 0$ for all $\alpha \in \bigoplus_{i \geq 0} \operatorname{Hom}_{R}\left(C, \Sigma^{i} C\right)$. Hence $p(t) \sim 0$, and thus $p\left(\operatorname{Hom}_{R}(t, D)\right)(\beta) \sim 0$ for all $\beta \in \bigoplus_{i \geq 0} \operatorname{Hom}_{R}\left(C, \Sigma^{i} D\right)$. Therefore $p(\chi) \in \operatorname{Ann} \bigoplus_{i \geq 0} \operatorname{Hom}_{R}\left(C, \Sigma^{i} D\right)$. Similarly if $p(\chi) \in \operatorname{Ann} \bigoplus_{i \geq 0} \operatorname{Hom}_{R}\left(D, \Sigma^{i} D\right)$ then $p(\chi) \in \operatorname{Ann} \bigoplus_{i \geq 0} \operatorname{Hom}_{R}\left(C, \Sigma^{i} D\right)$. Thus

$$
\begin{gathered}
\text { Ann } \bigoplus_{i \geq 0} \operatorname{Hom}_{R}\left(C, \Sigma^{i} C\right) \bigcap \operatorname{Ann} \bigoplus_{i \geq 0} \operatorname{Hom}_{R}\left(D, \Sigma^{i} D\right) \\
\subseteq \operatorname{Ann} \bigoplus_{i \geq 0} \operatorname{Hom}_{R}\left(C, \Sigma^{i} D\right)
\end{gathered}
$$

which implies that

$$
V(Q, f, C, D) \subseteq V(Q, f, C, C) \cap V(Q, f, D, D) .
$$

3.

$$
\begin{gathered}
V(Q, f, C, D)=V(Q, f, C, C) \cap V(Q, f, D, D)= \\
V(Q, f, C, C) \cap \mathbb{k}^{c}=V(Q, f, C, C)
\end{gathered}
$$

4. If $C=\bigoplus_{p=1}^{r} C_{p}$ and $D=\bigoplus_{q=1}^{s} D_{q}$, then we have an isomorphism of graded $R\left[\chi_{1}, \ldots, \chi_{c}\right]$ modules

$$
\bigoplus_{i \geq 0} \operatorname{Hom}_{R}\left(C, \Sigma^{i} D\right) \cong \bigoplus_{p, q} \bigoplus_{i \geq 0} \operatorname{Hom}_{R}\left(C_{p}, \Sigma^{i} D_{q}\right)
$$

and thus $V(Q, f, C, D)=\cup_{p, q} V\left(Q, f, C_{p}, D_{q}\right)$. 
5. Applying $\operatorname{Hom}_{Q_{a}}(\ldots, D)$ to the exact triangle

$$
T C \rightarrow T C^{\prime} \rightarrow T \text { Cone }(\alpha) \rightarrow \Sigma T C
$$

we get the following long exact sequence.

$$
\begin{aligned}
\cdots & \rightarrow \operatorname{Hom}_{Q_{a}}(\Sigma T C, T D) \rightarrow \operatorname{Hom}_{Q_{a}}(T \text { Cone }(\alpha), T D) \\
& \rightarrow \operatorname{Hom}_{Q_{a}}\left(T C^{\prime}, T D\right) \rightarrow \operatorname{Hom}_{Q_{a}}(T C, T D) \rightarrow \cdots
\end{aligned}
$$

If $\bar{a} \in \mathbb{k}$ is not in $V\left(Q, f, C^{\prime}, D\right) \cup V(Q, f$, Cone $(\alpha), D)$, then by Theorem 3.2.1, we have both $\operatorname{Hom}_{Q_{a}}\left(T C^{\prime}, \Sigma^{n} T D\right)=0$ and $\operatorname{Hom}_{Q_{a}}\left(T \operatorname{Cone}(\alpha), \Sigma^{n} T D\right)=$

0 for $n \gg 0$. Thus by the long exact sequence, $\operatorname{Hom}_{Q_{a}}\left(T C, \Sigma^{n} T D\right)=0$ for $n \gg 0$ and so $\bar{a} \notin V(Q, f, C, D)$. Hence we have

$$
V(Q, f, C, D) \subseteq V\left(Q, f, C^{\prime}, D\right) \cup V(Q, f, \text { Cone }(\alpha), D) .
$$

The other inclusions can be proved similarly.

\subsection{Rank Varieties of Higher Dimensions}

In [10], Bergh and Jorgensen generalize the definition of the rank variety for a pair of $R$-modules over an intermediate complete intersection of codimension $d$. In this section we will restate their results in $\mathrm{K}_{\mathrm{tac}}(R)$.

Let $I=\left(f_{1}, \ldots, f_{c}\right)$ and let $W$ be a subspace of the vector space $V=I / m I$. Consider the intermediate complete intersection $Q / J$ where the ideal $J \subseteq I$ is generated by a regular sequence obtained by taking preimages of a basis of $W$ [11]. The $k$-vector space $J / m J$ is isomorphic to $W$ by the map $\phi_{J}: J / m J \rightarrow W$ defined by $f+m J \rightarrow f+m I[10]$. Given two complete intersections $R^{\prime}=Q / J^{\prime}$ and $R^{\prime \prime}=Q / J^{\prime \prime}$, we call $R^{\prime}$ and $R^{\prime \prime}$ equivalent if $\phi_{j}(J / m J)=\phi_{J^{\prime}}\left(J^{\prime} / m J^{\prime}\right)$. 
Theorem 3.4.1. Let $W$ be a subspace of $V$ and consider the complete intersection $R^{\prime}=Q / J$ where $J$ is defined as above. Then for all $C, D \in \operatorname{Ktac}(R)$,

$$
\phi_{J}\left(W_{R^{\prime}}(Q, f T C, T D)\right)=V_{R}^{*}(Q, f, C, D) \cap W
$$

Theorem 3.4.2. Let $R^{\prime}=Q / J^{\prime}$ and $R^{\prime \prime}=Q / J^{\prime \prime}$ be two equivalent intermediate complete intersections. Then for all $C, D \in \mathrm{K}_{\mathrm{tac}}(R)$ we get

$$
\phi_{J}\left(V_{R^{\prime}}^{*}(Q, f, T C, T D)\right)=\phi_{J^{\prime}}\left(V_{R^{\prime \prime}}^{*}(Q, f, T C, T D)\right)
$$

Definition 3.4.3. Let $C, D \in \mathrm{K}_{\mathrm{tac}}(R)$ and let $W$ and $J$ be defined as above. Then we define the rank variety of dimension $d$ to be

$$
\begin{gathered}
W_{d}(Q, f, C, D)=\left\{p_{w} \in G_{d}(V) \mid \operatorname{Hom}_{Q / J}\left(\Sigma^{n} T C, T D\right)\right) \neq 0 \\
\text { for infinitely many } n>0\} \cup\{0\}
\end{gathered}
$$

where $G_{d}(V)$ is the Grassman variety of $d$-dimensional subspaces of $V$.

Note that if we consider $V^{*}$ to be a projective variety then $W(Q, f, C, D)=$ $W_{1}(Q, f, C, D)$.

Theorem 3.4.4. Let $Q / J$ and $Q / J^{\prime}$ be equivalent intermediate complete intersections. Then for all $C, D \in \mathrm{K}_{\mathrm{tac}}(R)$ we have $\operatorname{Hom}_{Q / J}(T C, T D)=0$ for all $i \gg 0$ if and only if $\operatorname{Hom}_{Q / J^{\prime}}(T C, T D)=0$ for all $i \gg 0$.

Theorem 3.4.5. For all complexes $C, D \in \mathrm{K}_{\mathrm{tac}}(R)$ we have that $W_{d}(Q, f, C, D)$ is a closed set in $G_{d}(V)$.

The proofs of these results are identical to those that Jorgensen and Bergh give for rank varieties of pairs of modules. 


\subsection{Examples}

We will now compute some simple examples of support varieties in $\mathrm{K}_{\mathrm{tac}}(R)$. In the first example we let the field be the complex numbers and choose a simple periodic complex $C$. The resulting support variety $V(Q, f, C, C)$ is a line in $\mathbb{C}^{2}$. Example 3.5.1. Let $R=\mathbb{C}[[x, y]] /\left(x^{2}, y^{2}\right)$ and $C$ be the complex

$$
\begin{gathered}
C: \cdots \stackrel{d_{n+1}^{C}}{\longrightarrow} R^{2} \stackrel{d_{n}^{C}}{\longrightarrow} R^{2} \stackrel{d_{n-1}^{C}}{\longrightarrow} R^{2} \stackrel{d_{n-2}^{C}}{\longrightarrow} \cdots \\
\text { where } d_{n}^{C}=\left(\begin{array}{cc}
x & y \sqrt{2} \\
y \sqrt{2} & -x
\end{array}\right) \text { for all } n .
\end{gathered}
$$

Then

$$
\tilde{d}^{2}=\left(\begin{array}{cc}
x^{2}+2 y^{2} & 0 \\
0 & x^{2}+2 y^{2}
\end{array}\right)=x^{2} \widetilde{t_{1}}+y^{2} \widetilde{t_{2}},
$$

and so we have

$$
t_{1}=\left(\begin{array}{cc}
1 & 0 \\
0 & 1
\end{array}\right) \text { and } t_{2}=\left(\begin{array}{cc}
2 & 0 \\
0 & 2
\end{array}\right)
$$

Thus

$$
\operatorname{Ann}\left(\operatorname{Hom}_{R}\left(C, \Sigma^{i} C\right) \otimes_{R} \mathbb{C}\right)=\left(\chi_{1}-2 \chi_{2}\right)
$$

and therefore

$$
V(Q, f, C, C)=Z\left(\chi_{1}-2 \chi_{2}\right)=\{(a, 2 a) \mid a \in \mathbb{C}\}
$$

The next example illustrates property 2 from Theorem 3.3.2, i.e. that

$$
V(Q, f, C, D)=V(Q, f, C, C) \cap V(Q, f, D, D)
$$

Example 3.5.2. Let $R=\mathbb{k}[[x, y, z]] /\left(x^{2}, y^{2}, z^{2}\right)$ and let $C$ be the complex

$$
C: \cdots \stackrel{d_{n+1}^{C}}{\longrightarrow} R^{2} \stackrel{d_{n}^{C}}{\longrightarrow} R^{2} \stackrel{d_{n-1}^{C}}{\longrightarrow} R^{2} \stackrel{d_{n-2}^{C}}{\longrightarrow} \cdots
$$




$$
\text { where } d_{n}^{C}=\left(\begin{array}{cc}
x & 0 \\
0 & y
\end{array}\right) \text { for all } n \text {. }
$$

Then

$$
\left(\tilde{d}^{C}\right)^{2}=\left(\begin{array}{cc}
x^{2} & 0 \\
0 & y^{2}
\end{array}\right)=x^{2} \widetilde{t_{1}}+y^{2} \widetilde{t_{2}}+z^{2} \widetilde{t_{3}},
$$

and so we have

$$
t_{1}=\left(\begin{array}{ll}
1 & 0 \\
0 & 0
\end{array}\right), t_{2}=\left(\begin{array}{ll}
0 & 0 \\
0 & 1
\end{array}\right) \text {, and } t_{3}=\left(\begin{array}{ll}
0 & 0 \\
0 & 0
\end{array}\right) .
$$

Therefore

$$
\operatorname{Ann}\left(\bigoplus_{i \geq 0} \operatorname{Hom}_{R}\left(C, \Sigma^{i} C\right) \otimes_{R} \mathbb{k}\right)=\left(\chi_{1} \chi_{2}, \chi_{3}\right),
$$

thus the support variety of $C$ is

$$
V(Q, f, C, C)=Z\left(\chi_{1} \chi_{2}, \chi_{3}\right)
$$

i.e. the union of the $x$ and $y$ axes.

Remark 3.5.3. Notice that $C=C_{1} \oplus C_{2}$ where

$$
C_{1}: \cdots \stackrel{x}{\longrightarrow} R \stackrel{x}{\longrightarrow} R \longrightarrow
$$

and

$$
C_{2}: \cdots \stackrel{y}{\longrightarrow} R \stackrel{y}{\longrightarrow} R \longrightarrow
$$

and so by property 4 of Theorem 3.3.2, we could simply compute the support varieties of $C_{1}$ and $C_{2}$ separately and take their union. The support variety of $C_{1}$ is the $x$ axis and the support variety of $C_{2}$ is the $y$ axis.

Now let $D$ be the complex

$$
D: \cdots \stackrel{d_{n+1}^{D}}{\longrightarrow} R^{2} \stackrel{d_{n}^{D}}{\longrightarrow} R^{2} \stackrel{d_{n-1}^{D}}{\longrightarrow} R^{2} \stackrel{d_{n-2}^{D}}{\longrightarrow} \cdots
$$




$$
\text { where } d_{n}^{D}=\left(\begin{array}{cc}
x & 0 \\
0 & z
\end{array}\right) \text { for all } n
$$

Then

$$
\left(\tilde{d}^{D}\right)^{2}=\left(\begin{array}{cc}
x^{2} & 0 \\
0 & z^{2}
\end{array}\right)=x^{2} \widetilde{s_{1}}+y^{2} \widetilde{s_{2}}+z^{2} \widetilde{s_{3}},
$$

and so

$$
s_{1}=\left(\begin{array}{cc}
1 & 0 \\
0 & 0
\end{array}\right), s_{2}=\left(\begin{array}{cc}
0 & 0 \\
0 & 0
\end{array}\right), \text { and } s_{3}=\left(\begin{array}{cc}
0 & 0 \\
0 & 1
\end{array}\right) .
$$

Therefore the support variety of $D$ is

$$
V(Q, f, D, D)=Z\left(\chi_{1} \chi_{3}, \chi_{2}\right)
$$

i.e. the union of the $x$ and $z$ axes.

When computing $V(Q, f, C, C)$ we can use the maps $t_{k}$ and $\operatorname{Hom}_{R}\left(t_{k}, C\right)$ interchangeably. Similarly we can use $s_{k}$ and $\operatorname{Hom}_{R}\left(s_{k}, D\right)$ interchangeably to compute $V(Q, f, D, D)$. However, to compute $V(Q, f, C, D)$ we need to determine either $\operatorname{Hom}_{R}\left(t_{k}, D\right)$ or $\operatorname{Hom}_{R}\left(C, s_{k}\right)$ for all $1 \leq k \leq c$. For any map $h \in \operatorname{Hom}_{R}\left(C, \Sigma^{i} D\right)$,

$$
h \circ\left(\begin{array}{ll}
x & 0 \\
0 & y
\end{array}\right)=\left(\begin{array}{ll}
x & 0 \\
0 & z
\end{array}\right) \circ h .
$$

This implies that $h$ is of the form $h_{n}=\left(\begin{array}{cc}a_{n} & 0 \\ 0 & 0\end{array}\right)$. By definition, $\operatorname{Hom}_{R}\left(t_{k}, C\right)(h)=$ $h \circ t_{k}$ so now have $\operatorname{Hom}_{R}\left(t_{1}, D\right)=\left(\begin{array}{ll}1 & 0 \\ 0 & 0\end{array}\right)$ and $\operatorname{Hom}_{R}\left(t_{2}, D\right)=\operatorname{Hom}_{R}\left(t_{3}, D\right)=$ $\left(\begin{array}{ll}0 & 0 \\ 0 & 0\end{array}\right)$. Therefore

$$
\operatorname{Ann}\left(\operatorname{Hom}_{R}\left(C, \Sigma^{i} D\right) \otimes_{R} \mathbb{k}\right)=\left(\chi_{2}, \chi_{3}\right)
$$


so we get that

$$
V(Q, f, C, D)=Z\left(\chi_{2}, \chi_{3}\right)=V(Q, f, C, C) \cap V(Q, f, D, D)
$$

i.e. the support variety of $C$ and $D$ is the $x$ axis. 


\section{Chapter 4}

\section{Realizability}

Our goal in this chapter is to prove that any homogeneous affine variety is realizable as the support of some complex in the category of totally acyclic chain complexes, $\mathrm{K}_{\mathrm{tac}}(R)$, over a complete intersection ring, $R=Q /\left(f_{1}, \ldots, f_{c}\right)$. Petter Bergh [8] showed that it is possible to construct a module over a complete intersection ring with any desired support variety. Avramov and Iyengar [4] proved a similar realizability result in the derived category.

By a theorem of Buchweitz [12], the triangulated category $\mathrm{K}_{\mathrm{tac}}(R)$ over any Gorenstein ring is equivalent to the singularity category, i.e. the derived category modulo the perfect complexes. The support variety of any perfect complex is $\{0\}$ $[7,11.6]$. Thus support varieties are well defined in the singularity category and so we could define the support variety of a pair of objects in $\mathrm{K}_{\text {tac }}(R)$ via the Buchweitz equivalence. We could then use the same argument Avramov and Iyengar use to prove realizability in $\mathrm{K}_{\mathrm{tac}}(R)$. However, we would prefer a more direct proof of realizability in this setting.

Starting with a complete resolution of the residue field, we construct a sequence of totally acyclic complexes. Each complex is a mapping cone of a polynomial of the Eisenbud operators from the previous complex. The support variety of the final mapping cone is the zero set of this sequence of polynomials. Thus we can explicitly create a totally acyclic complex with a desired support.

During the proof of realizability of support, we encounter an interesting question. Consider a complex $C$ with Eisenbud operators $t_{k}$ and let $p$ be a polynomial 
such that $p\left(t_{k}\right) \sim 0$. Now consider the mapping cone Cone $(p)$ and its Eisenbud operators $s_{k}$. In order to show that the mapping cone has the desired support, we would like to show that $p\left(s_{k}\right) \sim 0$. However, we are only able to show that $p^{2}\left(s_{k}\right) \sim 0$. This is sufficient since the algebraic variety of an ideal is equal to the variety of the radical of that ideal. However, we are also interested in investigating the scheme structure of the support.

Interestingly for all of the simple examples of mapping cones we have computed, if $p\left(t_{k}\right) \sim 0$, then $p\left(s_{k}\right) \sim 0$. We present a method for constructing totally acyclic complexes with nilpotent Eisenbud operators by tensoring a known totally acyclic complex with a perfect complex. However, we do not know of an example of such a complex which is also a mapping cone of a polynomial of Eisenbud operators.

\subsection{Constructing a Sequence of Mapping Cones}

The main goal of this section is to prove that for any cone $\mathcal{V}$ in $\mathbb{k}^{c}$, there exists some complex $C \in \mathrm{K}_{\mathrm{tac}}(R)$ such that $V(Q, f, C, C)=\mathcal{V}$. Any variety $\mathcal{V} \in \mathbb{k}^{C}$ is the zero set $Z\left(p_{1}, \ldots, p_{m}\right)$ for some list of polynomials $p_{1}, \ldots, p_{m}$. Thus our goal is to construct a complex $C$ with cohomology operators $\left\{t_{j} \mid 1 \leq j \leq c\right\}$ such that $p_{k}(t) \sim 0$ for all $1 \leq k \leq m$ and so that for any polynomial $q \notin\left(p_{1}, \ldots, p_{m}\right), q \nsim 0$. Starting with a complete resolution of the residue field $\mathbb{k}$, we will construct $C$ inductively by taking a sequence of mapping cones of the $p_{k}^{\prime} s$. In particular, we will show that if $V(Q, f, C, C)=Z\left(p_{1}, \ldots, p_{m}\right)$, then $V\left(Q, f, \operatorname{Cone}\left(p_{m+1}\right)\right.$, Cone $\left.\left(p_{m+1}\right)\right)=$ $Z\left(p_{1}, \ldots, p_{m}, p_{m+1}\right)$. To prove this, we need to show three facts:

1. For any $p \in R\left[\chi_{1}, \ldots, \chi_{c}\right], p \in \operatorname{Ann} \bigoplus_{i \geq 0} \operatorname{Hom}_{R}\left(\operatorname{Cone}(p), \Sigma^{i} \operatorname{Cone}(p)\right)$.

2. For any $p, q \in R\left[\chi_{1}, \ldots, \chi_{c}\right]$, if $q \in \operatorname{Ann} \bigoplus_{i \geq 0} \operatorname{Hom}_{R}\left(C, \Sigma^{i} C\right)$ then $q^{2} \in \operatorname{Ann} \bigoplus_{i \geq 0} \operatorname{Hom}_{R}\left(\operatorname{Cone}(p), \Sigma^{i} \operatorname{Cone}(p)\right)$. 
3. For any $p(\chi) \in R\left[\chi_{1}, \ldots, \chi_{c}\right]$,

$$
\sqrt{\operatorname{Ann} \bigoplus_{i \geq 0} \operatorname{Hom}_{R}\left(\operatorname{Cone}(p), \Sigma^{i} \operatorname{Cone}(p)\right)} \subseteq \sqrt{\operatorname{Ann} \bigoplus_{i \geq 0} \operatorname{Hom}_{R}\left(C, \Sigma^{i} C\right)} .
$$

These three properties can be roughly summarized as (1) the new polynomial is zero, (2) the old polynomials are still zero, and (3) no extra polynomials are zero.

\section{Property 1: The New Polynomial}

To prove (1), we will let $p$ be any polynomial of cohomology operators of a complex $C$. We will then show that the same polynomial applied to the cohomology operators of Cone $(P)$ will always be homotopic to zero.

Lemma 4.1.1. Let $R=Q /\left(f_{1}, \ldots, f_{c}\right)$ be a complete intersection ring and let $C \in$ $\mathrm{K}_{\mathrm{tac}}(R)$. If $p$ is any homogeneous polynomial of cohomology operators, then $p \in$ Ann $\underset{i \geq 0}{\bigoplus} \operatorname{Hom}_{R}\left(\operatorname{Cone}(p), \Sigma^{i} \operatorname{Cone}(p)\right)$

Proof. Lift the complex $C$ to $Q$ so that $\tilde{d}^{2}=\sum_{j=1}^{c} f_{j} \tilde{t}_{j}$. Let $p(t)=\sum_{\alpha} q_{\alpha} t^{\alpha}$ where $q_{\alpha} \in R$ and $t^{\alpha}=t_{1}^{\alpha_{1}} \cdots t_{c}^{\alpha_{c}}$ is a monomial in the $t_{j}^{\prime} s$. Now consider the complex Cone $(p)$ with differentials

$$
d^{\text {Cone }(t)}=\left(\begin{array}{cc}
d^{\Sigma C} & 0 \\
\sum_{\alpha} q_{\alpha} t^{\alpha}\left(\sigma_{C}^{1}\right)^{-1} & d^{\Sigma^{2 r} C}
\end{array}\right)
$$

where $r=\operatorname{deg} p$. We can now lift the differential to get

$$
\tilde{d}^{\text {Cone }(t)}=\left(\begin{array}{cc}
\tilde{d}^{\Sigma C} & 0 \\
\sum_{\alpha} q_{\alpha} \tilde{\sigma}_{C}^{\alpha} w^{\alpha}\left(\tilde{\sigma}_{C}^{1}\right)^{-1} & \tilde{d}^{\Sigma^{2 r} C}
\end{array}\right)
$$

For simplicity of notation, we will let $w^{\alpha}$ be the appropriate composition of $\tilde{t}$ 's and $\sigma$ 's such that $w^{\alpha} \otimes_{Q} R=t^{\alpha}$, i.e.

$$
w^{\alpha}=\tilde{\sigma}_{\Sigma^{2 \alpha-2}}^{2} \tilde{t}_{1} \tilde{\sigma}_{\Sigma^{2 \alpha-4}}^{2} \tilde{t}_{1} \cdots \tilde{\sigma}_{C}^{2} \tilde{t}_{c}
$$


Squaring the differential of the cone yields

$$
\left(\tilde{d}^{\text {Cone }(t)}\right)^{2}=\left(\begin{array}{cc}
\left(\tilde{d}^{\Sigma^{2} C}\right)^{2} & 0 \\
\sum_{\alpha} q_{\alpha} A_{\alpha} & \left(\tilde{d}^{\Sigma^{2 d}}\right)^{2}
\end{array}\right)=\sum_{j=1}^{c} f_{j} \widetilde{s_{j}}
$$

where

$$
A_{\alpha}=\tilde{d}^{\Sigma^{2 r} C} w^{\alpha}\left(\tilde{\sigma}_{C}^{1}\right)^{-1}+w^{\alpha}\left(\tilde{\sigma}_{C}^{1}\right)^{-1} \tilde{d}^{\Sigma C}
$$

Remark 4.1.2. Recalling the sign convention from $d^{\Sigma^{i} C}=(-1)^{i}\left(\sigma_{C}^{i}\right) d^{C}\left(\sigma_{C}^{i}\right)^{-1}$, one can see that $A_{\alpha}$ measures the non-commutativity of $\tilde{d}$ and $w^{\alpha}$

We can write the $\tilde{s}_{j}$ 's as

$$
\widetilde{s_{j}}=\left(\begin{array}{cc}
\tilde{\sigma}_{C}^{2} \widetilde{t_{j}} & 0 \\
\sum_{\alpha} q_{\alpha} a_{j, \alpha} & \tilde{\sigma}_{C}^{2} \widetilde{t_{j}}
\end{array}\right)\left(\tilde{\sigma}_{C}^{1}\right)^{-1} .
$$

where the $a_{j, \alpha}$ 's are determined by decomposing the $A_{\alpha}$ 's, i.e.

$$
A_{\alpha}=\sum_{j=1}^{c} f_{j} a_{j, \alpha}
$$

To show that $p \in$ Ann $\bigoplus_{i \geq 0} \operatorname{Hom}_{R}\left(\operatorname{Cone}(p), \Sigma^{i} \operatorname{Cone}(p)\right)$, we need to show that $p(s)=$ $p(\tilde{s}) \otimes_{Q} R$ is homotopic to zero. In general, $p(\tilde{s})$ is of the form

$$
p(\tilde{s})=\sum_{\alpha} \tilde{s}^{\alpha}=\sum_{\alpha}\left(\begin{array}{cc}
w^{\alpha} & 0 \\
b_{\alpha} & w^{\alpha}
\end{array}\right)\left(\tilde{\sigma}_{C}^{1}\right)^{-1}
$$

where $b_{\alpha}$ can be written in terms of the $a_{j, \alpha}$ 's. In particular,

$$
b_{\alpha}=\sum_{\beta+\gamma=\alpha-1} w^{\beta} q_{\alpha} a_{j, \alpha} w^{\gamma}
$$

where

$$
w^{\beta}=\tilde{\sigma}_{\Sigma^{2 \alpha-2}}^{2} \tilde{t}_{1} \tilde{\sigma}_{\Sigma^{2 \alpha-4}}^{2} \tilde{t}_{1} \cdots \tilde{t}_{j-1} \text { and } w^{\gamma}=\tilde{\sigma}_{\Sigma^{2 \gamma-2}}^{2} \tilde{t}_{j+1} \cdots \tilde{\sigma}_{C}^{2} \tilde{t}_{c} .
$$


We need to show that $b_{\alpha}$ is homotopic to zero for any $\alpha$. Thus we need to understand the $a_{j, \alpha}$ 's by decomposing $A_{\alpha}$. We can rewrite $A_{\alpha}$ from equation 4.1.1.1 by adding zero repeatedly to see that

$$
A_{\alpha}=\tilde{d}^{\Sigma^{2 r} C} w^{\alpha}\left(\tilde{\sigma}_{C}^{1}\right)^{-1}+w^{\alpha}\left(\tilde{\sigma}_{C}^{1}\right)^{-1} \tilde{d}^{\Sigma C}
$$

$$
=\sum_{j, e} w^{\beta} \tilde{t}_{j}^{e}\left(\tilde{\sigma}_{C}^{2 r}\right)^{-1}\left(\tilde{d}^{\Sigma^{2 r} C} \tilde{\sigma}_{C}^{2 r} \tilde{t}_{j}\left(\tilde{\sigma}_{C}^{1}\right)^{-1}+\tilde{\sigma}_{C}^{2 r} \tilde{t}_{j}\left(\tilde{\sigma}_{C}^{1}\right)^{-1} \tilde{d}^{\Sigma C}\right) \tilde{\sigma}_{C}^{\gamma+\alpha_{j}-k} \tilde{t}_{j}^{\alpha_{j}-e} w^{\gamma} \text {. }
$$

This allows us to focus on the term

$$
\left(\tilde{d}^{\Sigma^{2 r} C} \tilde{\sigma}_{C}^{2 r} \tilde{t}_{j}\left(\tilde{\sigma}_{C}^{1}\right)^{-1}+\tilde{\sigma}_{C}^{2 r} \tilde{t}_{j}\left(\tilde{\sigma}_{C}^{1}\right)^{-1} \tilde{d}^{\Sigma C}\right)
$$

which is in terms of only $\tilde{t}_{j}$ to the first power rather than $w^{\alpha}$ which is a product of $t_{j}$ 's to various powers. Since

$$
\left(\tilde{d}^{\Sigma^{2 r} C}\right)^{3} \tilde{\sigma}_{C}^{2 r}\left(\tilde{\sigma}_{C}^{1}\right)^{-1}+\tilde{\sigma}_{C}^{2 r}\left(\tilde{\sigma}_{C}^{1}\right)^{-1}\left(\tilde{d}^{\Sigma C}\right)^{3}=0
$$

we get that

$$
\tilde{d}^{\Sigma^{2 r} C}\left(\sum_{j=1}^{c} f_{j} \tilde{\sigma}_{C}^{2 r} \tilde{t}_{j}\left(\tilde{\sigma}_{C}^{1}\right)^{-1}\right)+\left(\sum_{j=1}^{c} f_{j} \tilde{\sigma}_{C}^{2 r} \tilde{t}_{j}\left(\tilde{\sigma}_{C}^{1}\right)^{-1}\right) \tilde{d}^{\Sigma C}=0 .
$$

We can rewrite this as

$$
\begin{aligned}
& f_{k}\left(\tilde{d}^{\Sigma^{2 r} C} \tilde{\sigma}_{C}^{2 r} \tilde{t}_{k}\left(\tilde{\sigma}_{C}^{1}\right)^{-1}+\tilde{\sigma}_{C}^{2 r} \tilde{t}_{k}\left(\tilde{\sigma}_{C}^{1}\right)^{-1} \tilde{d}^{\Sigma C}\right)= \\
& \sum_{j \neq k}^{c} f_{j}\left(\tilde{d}^{\Sigma^{2 r} C} \tilde{\sigma}_{C}^{2 r} \tilde{t}_{j}\left(\tilde{\sigma}_{C}^{1}\right)^{-1}+\tilde{\sigma}_{C}^{2 r} \tilde{t}_{j}\left(\tilde{\sigma}_{C}^{1}\right)^{-1} \tilde{d}^{\Sigma C}\right)
\end{aligned}
$$

for any $1 \leq k \leq c$. Hence

$$
\tilde{d}^{\Sigma^{2 r} C} \tilde{\sigma}_{C}^{2 r} \tilde{t}_{k}\left(\tilde{\sigma}_{C}^{1}\right)^{-1}+\tilde{\sigma}_{C}^{2 r} \tilde{t}_{k}\left(\tilde{\sigma}_{C}^{1}\right)^{-1} \tilde{d}^{\Sigma C} \in\left(f_{1}, \ldots, \hat{f}_{k}, \ldots, f_{c}\right) \operatorname{Hom}\left(C, \Sigma^{i} C\right)
$$

for each $k$. Thus we can write it as

$$
\tilde{d}^{\Sigma^{2 r} C} \tilde{\sigma}_{C}^{2 r} \tilde{t}_{j}\left(\tilde{\sigma}_{C}^{1}\right)^{-1}+\tilde{\sigma}_{C}^{2 r} \tilde{t}_{j}\left(\tilde{\sigma}_{C}^{1}\right)^{-1} \tilde{d}^{\Sigma C}=\sum_{j \neq k} f_{j} \tilde{\tau}_{j_{k}}
$$


We can now substitute the $\tilde{\tau}$ 's from 4.1.2.4 into 4.1.2.3 to get

$$
\sum_{k=1}^{c} f_{k} \sum_{j \neq k} f_{j} \tilde{\tau}_{j_{k}}=0
$$

which can be rearranged into

$$
\sum_{j \neq k}^{c} f_{j} f_{k}\left(\tilde{\tau}_{j_{k}}+\tilde{\tau}_{j_{k}}\right)=0
$$

But this means that $\left(\tilde{\tau}_{j_{k}}+\tilde{\tau}_{k_{j}}\right) \in\left(f_{1}, \ldots, f_{c}\right) \operatorname{Hom}_{R}\left(C, \Sigma^{i} C\right)$. Hence $\left(\tilde{\tau}_{j_{k}}+\tilde{\tau}_{k_{j}}\right) \otimes_{Q}$ $R \sim 0$.

Now let us return to considering $a_{j, \alpha}$, the bottom left corner of $s_{j}$. Substituting in the definition of the $\tilde{\tau}_{k}$ 's from 4.1.2.4 into equation 4.1.2.2 we get

$$
\begin{gathered}
\sum_{j=1}^{c} f_{j} a_{j, \alpha}=A_{\alpha}=\tilde{d}^{\Sigma^{2 r} C} w^{\alpha}\left(\tilde{\sigma}_{C}^{1}\right)^{-1}+w^{\alpha}\left(\tilde{\sigma}_{C}^{1}\right)^{-1} \tilde{d}^{\Sigma C}= \\
\sum_{j, e} w^{\beta} \tilde{t}_{j}^{e}\left(\tilde{\sigma}_{C}^{2 r}\right)^{-1}\left(\sum_{k \neq j} f_{k} \tilde{\tau}_{k_{j}}\right) \tilde{\sigma}_{C}^{\gamma+\alpha_{j}-k} \tilde{t}_{j}^{\alpha_{j}-e} w^{\gamma}
\end{gathered}
$$

Thus we can see that

$$
a_{j, \alpha}=\sum_{e} \sum_{k \neq j} w^{\alpha} \tilde{t}_{j}^{e}\left(\tilde{\sigma}_{C}^{2 r}\right)^{-1}\left(\tilde{\tau}_{k_{j}}\right) \tilde{\sigma}_{C}^{\gamma+\alpha_{j}-k} \tilde{t}_{j}^{n-e} w^{\beta}
$$

Substituting $a_{j, \alpha}$ from 4.1.2.6 into equation 4.1.2.1 and rearranging the terms we see that

$$
b_{\alpha}=\sum_{j, k} w^{2 \beta} \tilde{t}_{j}^{n_{j}} q_{\alpha}\left(\tilde{\tau}_{j_{k}}+\tilde{\tau}_{k_{j}}\right) \tilde{t}_{j}^{n_{j}} w^{2 \gamma}
$$

Recall from above that $\left(\tilde{\tau}_{j_{k}}+\tilde{\tau}_{k_{j}}\right) \otimes_{Q} R \sim 0$ and thus $b_{\alpha}$ is homotopic to zero when tensored down to $R$. So now $p(s)=p(\tilde{s}) \otimes_{Q} R=\sum_{\alpha}\left(\begin{array}{cc}t^{\alpha} & 0 \\ 0 & t^{\alpha}\end{array}\right)$. Taking the homotopy map $\lambda=\left(\begin{array}{ll}0 & 1 \\ 0 & 0\end{array}\right)$ we get
\[ \quad \lambda \circ d^{\operatorname{Cone}(p)}+d^{\operatorname{Cone}(p)} \circ \lambda=p(s) . \]

Thus $p(s) \sim 0$. 
Property 2: The Old Polynomials

We would now like to show that if a polynomial $q$ of cohomology operators is in Ann $\bigoplus_{i \geq 0} \operatorname{Hom}_{R}\left(C, \Sigma^{i} C\right)$, then $q \in \operatorname{Ann} \bigoplus_{i \geq 0} \operatorname{Hom}_{R}\left(\operatorname{Cone}(p), \Sigma^{i} \operatorname{Cone}(p)\right)$ for any polynomial $p$ of Eisenbud operators. Unfortunately we can only expect that $q^{2}$ will be in Ann $\bigoplus_{i \geq 0} \operatorname{Hom}_{R}\left(\operatorname{Cone}(p), \Sigma^{i} \operatorname{Cone}(p)\right)$. However this will be sufficient for our purposes since by Hilbert's Nullstellensatz, the variety of an ideal $I$ is equal to the variety of $\sqrt{I}$.

Lemma 4.1.3. Let $p, q \in R\left[\chi_{1}, \ldots, \chi_{c}\right]$. If $q \in \operatorname{Ann} \bigoplus_{i \geq 0} \operatorname{Hom}_{R}\left(C, \Sigma^{i} C\right)$ then $q^{2} \in$ Ann $\underset{i \geq 0}{\bigoplus_{1}} \operatorname{Hom}_{R}\left(\operatorname{Cone}(p), \Sigma^{i} \operatorname{Cone}(p)\right)$.

Proof. Let $q(\chi) \in \operatorname{Ann} \bigoplus_{i \geq 0} \operatorname{Hom}_{R}\left(C, \Sigma^{i} C\right)$. Then $q(t) \sim 0$, i.e. there exist homotopy maps $\lambda$ such that $q(t)=\lambda \circ d^{\Sigma C}+d^{\Sigma^{\operatorname{deg} p} C} \circ \lambda$. Applying the polynomial $q$ to the Eisenbud operators of the cone we see that $q(s)$ is of the form $q(s)=\left(\begin{array}{cc}q(t) & 0 \\ b & q(t)\end{array}\right)$. However,

$$
\begin{gathered}
\left(\begin{array}{cc}
q(t) & 0 \\
b & q(t)
\end{array}\right)-\left(\begin{array}{cc}
0 & 0 \\
b & 0)
\end{array}\right)=\left(\begin{array}{cc}
q(t) & 0 \\
0 & q(t)
\end{array}\right) \\
=\left(\begin{array}{cc}
d^{\Sigma C} & 0 \\
p(t) & d^{\Sigma^{\operatorname{deg} p} C}
\end{array}\right)\left(\begin{array}{ll}
\lambda & 0 \\
0 & 0
\end{array}\right)+\left(\begin{array}{cc}
d^{\Sigma C} & 0 \\
p(t) & d^{\Sigma^{\operatorname{deg} p} C}
\end{array}\right)\left(\begin{array}{ll}
0 & 0 \\
0 & \lambda
\end{array}\right) .
\end{gathered}
$$

Hence $q(s) \sim\left(\begin{array}{ll}0 & 0 \\ b & 0\end{array}\right)$ and $\left(\begin{array}{ll}0 & 0 \\ b & 0\end{array}\right)^{2}=\left(\begin{array}{ll}0 & 0 \\ 0 & 0\end{array}\right)$. Thus $q(s)^{2} \sim 0$ and therefore $q(\chi)^{2} \in \operatorname{Ann} \underset{i \geq 0}{\bigoplus} \operatorname{Hom}_{R}\left(\operatorname{Cone}(p), \Sigma^{i} \operatorname{Cone}(p)\right)$.

Property 3: No Extra Polynomials

Next we will show (3), that for any polynomial $p$ of Eisenbud operators,

$$
\sqrt{\operatorname{Ann} \bigoplus_{i \geq 0} \operatorname{Hom}_{R}\left(\operatorname{Cone}(p), \Sigma^{i} \operatorname{Cone}(p)\right)} \subseteq \sqrt{\operatorname{Ann} \bigoplus_{i \geq 0} \operatorname{Hom}_{R}\left(C, \Sigma^{i} C\right)} .
$$


This shows that the support variety of the mapping cone will have no unwanted defining polynomials.

Lemma 4.1.4. Let $(A, \mathrm{~m}, \mathbb{k})$ be a local ring and

$$
\cdots \longrightarrow X_{i} \longrightarrow Y_{i} \stackrel{p}{\longrightarrow} Y_{i-1} \longrightarrow X_{i-1} \longrightarrow \cdots
$$

be an exact sequence of $A$-modules where $p \in \mathrm{m}, X=\bigoplus_{i} X_{i}$, and $Y=\bigoplus_{i} Y_{i}$. Then $\sqrt{\operatorname{Ann} X} \subseteq \sqrt{(\operatorname{Ann} Y, p)}$.

Proof. Let $I$ be a prime ideal containing $p$ but not containing $\sqrt{\operatorname{Ann} X}$. Localizing at $I$ gives us $X_{I}=0$ and therefore we have the exact sequence

$$
0 \longrightarrow Y_{I_{i}} \stackrel{p}{\longrightarrow} Y_{I_{i-1}} \longrightarrow 0 \text {. }
$$

Therefore $Y_{I}=p Y_{I}$. Since $p$ is an element of the maximal ideal of $A$, Nakayama's lemma implies that $Y_{I}=0$ and hence $I$ does not contain $\sqrt{\operatorname{Ann} Y}$. Hence $\sqrt{\operatorname{Ann} X} \subseteq$ $\sqrt{(\operatorname{Ann} Y, p)}$.

Lemma 4.1.5. Let $C \in \mathrm{K}_{\mathrm{tac}}(R)$ and let $p(\chi) \in R\left[\chi_{1}, \ldots, \chi_{c}\right]$ be a polynomial of cohomology operators. Then

$$
\sqrt{\operatorname{Ann} \bigoplus_{i \geq 0} \operatorname{Hom}_{R}\left(\operatorname{Cone}(p), \Sigma^{i} \operatorname{Cone}(p)\right)} \subseteq \sqrt{\operatorname{Ann} \bigoplus_{i \geq 0} \operatorname{Hom}_{R}\left(C, \Sigma^{i} C\right)} .
$$

Proof. Consider the exact triangle

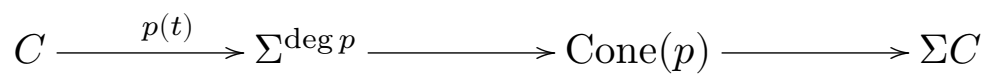

Applying $\operatorname{Hom}(\ldots$, Cone $(p))$ to the above triangle we get the long exact sequence

$$
\begin{gathered}
\cdots \rightarrow \operatorname{Hom}_{R}\left(\Sigma^{i} \operatorname{Cone}(p), \operatorname{Cone}(p)\right) \rightarrow \operatorname{Hom}_{R}\left(\Sigma^{i+\operatorname{deg} p} C, \operatorname{Cone}(p)\right) \rightarrow \\
\operatorname{Hom}_{R}\left(\Sigma^{i} C, \operatorname{Cone}(p)\right) \rightarrow \operatorname{Hom}_{R}\left(\Sigma^{i-1} \operatorname{Cone}(p), \operatorname{Cone}(p)\right) \rightarrow \cdots .
\end{gathered}
$$


Now recall that $\bigoplus_{i \leq 0} \operatorname{Hom}_{R}\left(\Sigma^{-i} C, C\right)=\bigoplus_{i \geq 0} \operatorname{Hom}_{R}\left(C, \Sigma^{i} C\right)$ and so we can apply Lemma 4.1.4 to see that

$$
\sqrt{\operatorname{Ann} \bigoplus_{i \geq 0} \operatorname{Hom}_{R}\left(\operatorname{Cone}(p), \Sigma^{i} \operatorname{Cone}(p)\right)} \subseteq \sqrt{\operatorname{Ann} \bigoplus_{i \geq 0} \operatorname{Hom}_{R}\left(C, \Sigma^{i} \operatorname{Cone}(p)\right)} .
$$

Now applying $\operatorname{Hom}\left(C,{ }_{-}\right)$to the exact triangle we get the long exact sequence

$$
\begin{gathered}
\cdots \rightarrow \operatorname{Hom}_{R}\left(\operatorname{Cone}(p), \Sigma^{i-1} C\right) \rightarrow \operatorname{Hom}_{R}\left(C, \Sigma^{i} C\right) \rightarrow \\
\operatorname{Hom}_{R}\left(C, \Sigma^{i+\operatorname{deg} p} C\right) \rightarrow \operatorname{Hom}_{R}\left(C, \Sigma^{i} \operatorname{Cone}(p)\right) \rightarrow \cdots
\end{gathered}
$$

Once again applying Lemma 4.1.4, we get that

$$
\sqrt{\operatorname{Ann} \bigoplus_{i \geq 0} \operatorname{Hom}_{R}(\operatorname{Cone}(p), C)} \subseteq \sqrt{\operatorname{Ann} \bigoplus_{i \geq 0} \operatorname{Hom}_{R}\left(C, \Sigma^{i} C\right)} .
$$

Therefore we now have

$$
\sqrt{\operatorname{Ann} \bigoplus_{i \geq 0} \operatorname{Hom}_{R}\left(\operatorname{Cone}(p), \Sigma^{i} \operatorname{Cone}(p)\right)} \subseteq \sqrt{\operatorname{Ann} \bigoplus_{i \geq 0} \operatorname{Hom}_{R}\left(C, \Sigma^{i} C\right)} .
$$

Realizing an Affine Variety

Putting the previous lemmas together, we get the following result.

Theorem 4.1.6. Let $R=Q /\left(f_{1}, \ldots, f_{c}\right)$ be a complete intersection ring and let $C \in$ $\mathrm{K}_{\mathrm{tac}}(R)$ with $V(Q, f, C, C)=Z\left(p_{1}, \ldots, p_{m}\right)$. Then for any homogeneous polynomial $p_{m+1}$ of cohomology operators,

$$
V\left(Q, f, \text { Cone }\left(p_{m+1}\right), \operatorname{Cone}\left(p_{m+1}\right)\right)=Z\left(p_{1}, \ldots, p_{m}, p_{m+1}\right)
$$

Proof. By Lemma 4.1.1, $p_{m+1} \in \operatorname{Ann} \underset{i \geq 0}{\bigoplus} \operatorname{Hom}\left(\operatorname{Cone}\left(p_{m+1}\right), \Sigma^{i} \operatorname{Cone}\left(p_{m+1}\right)\right)$. By Lemma 4.1.3, $p_{k}^{2} \in \operatorname{Ann} \bigoplus_{i \geq 0} \operatorname{Hom}\left(\operatorname{Cone}\left(p_{m+1}\right), \Sigma^{i} \operatorname{Cone}\left(p_{m+1}\right)\right)$ for all $1 \leq k \leq m$. Also by Lemma 4.1.5, if $q^{r} \in \operatorname{Ann} \underset{i \geq 0}{\bigoplus} \operatorname{Hom}\left(\operatorname{Cone}\left(p_{m+1}\right), \Sigma^{i} \operatorname{Cone}\left(p_{m+1}\right)\right)$ then $q \in$ 


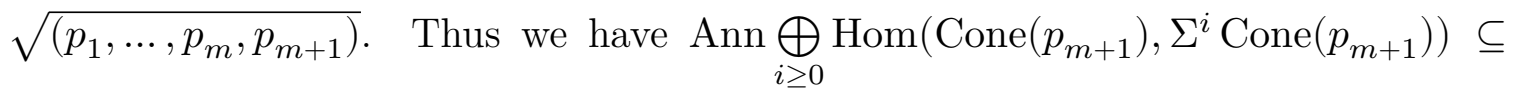
$\sqrt{\left(p_{1}, \ldots, p_{m}, p_{m+1}\right)}$. Therefore

$V\left(Q, f, \operatorname{Cone}\left(p_{m+1}\right), \operatorname{Cone}\left(p_{m+1}\right)\right)=Z\left(\sqrt{\left(p_{1}, \ldots, p_{m}, p_{m+1}\right)}\right)=Z\left(p_{1}, \ldots, p_{m}, p_{m+1}\right)$

We now have the tools needed to prove our main result.

Theorem 4.1.7. For any cone $\mathcal{V} \in \mathbb{k}^{c}$, there exists a complex $C \in \mathrm{K}_{\mathrm{tac}}(R)$ such that $V(Q, f, C, C)=\mathcal{V}$

Proof. Let $\mathcal{V}_{m}=Z\left(p_{1}, \ldots, p_{m}\right)$ be a cone in $\mathbb{k}^{c}$. We will now construct $C$ inductively. First consider $\mathcal{V}_{0}=Z(0)=\mathbb{k}^{c}$. If $D$ is a complete resolution of the field $\mathbb{k}$, then by Theorem 3.3.2 $V(Q, f, D, D)=\mathcal{V}$. Now assume that there exists $F \in \mathrm{K}_{\text {tac }}(R)$ such that $V(Q, f, F, F)=Z\left(p_{1}, \ldots, p_{m-1}\right)$. Let $C=\operatorname{Cone}\left(p_{m+1}\right)$. Then by Theorem 4.1.6,

$$
V(Q, f, C, C)=Z\left(p_{1}, \ldots, p_{m}\right)
$$

\subsection{Nilpotent Eisenbud Operators}

In the previous section, we took advantage of the nullstellensatz to prove that cones in $\mathbb{k}^{c}$ are realizable as the support of a totally acyclic complex. In particular, if a non-zero polynomial $p$ of Eisenbud operators is nilpotent, then $p$ is a defining polynomial of the support variety. Thus nilpotent Eisenbud operators contribute nothing to the support variety of a complex. However, we are interested in understanding how the support variety of a totally acyclic complex differs from its scheme structure. 
In this section we will provide a method for creating totally acyclic complexes with nilpotent Eisenbud operators.

Consider a totally acyclic complex $C$ and a perfect complex $P$, i.e. $P$ is a bounded complex of finitely generated projective modules,

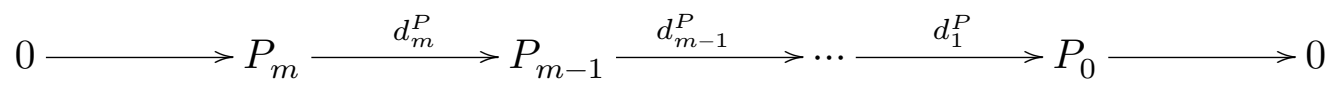

such that $\left(\tilde{d}^{C}\right)^{2}=\sum_{k=1}^{c} f_{k} \tilde{t}_{k}$ and $\left(\tilde{d}^{P}\right)^{2}=\sum_{k=1}^{c} f_{c} \tilde{r}_{k}$. Let $\Delta_{n, j}^{C}=d_{n-j}^{C} \otimes P_{j}$ and $\Delta_{n, j}^{P}=(-1)^{n-j} C_{n-j} \otimes d_{j}^{P}$. Since $C$ is totally acyclic and the $P_{i}^{\prime}$ 's are free and therefore flat, the bi-complex $C \otimes_{R} P$ has exact rows. Therefore the acyclic assembly lemma $[22,2.7 .3]$ implies the total complex $\operatorname{Tot}^{\oplus}\left(C \otimes_{R} P\right)$ is totally acyclic. It has differentials of the form

$$
d_{n}^{C \otimes_{R} P}=\left(\begin{array}{ccccc}
\Delta_{n, m}^{C} & \Delta_{n, m}^{P} & 0 & \ldots & 0 \\
0 & \Delta_{n, m-1}^{C} & \Delta_{n, m-1}^{P} & \ldots & 0 \\
\vdots & \ddots & & & \vdots \\
0 & \ldots & 0 & \Delta_{n, 1}^{C} & \Delta_{n, 1}^{P} \\
0 & \ldots & 0 & 0 & \Delta_{n, 0}^{C}
\end{array}\right) .
$$

Lifting this matrix to $Q$ and squaring it yields

$$
\left(\tilde{d}^{C \otimes_{R} P}\right)^{2}=\left(\begin{array}{cccccc}
\left(\widetilde{\Delta}^{C}\right)^{2} & 0 & \left(\widetilde{\Delta}^{P}\right)^{2} & 0 & \cdots & 0 \\
0 & \left(\widetilde{\Delta}^{C}\right)^{2} & 0 & \left(\widetilde{\Delta}^{P}\right)^{2} & \cdots & 0 \\
\vdots & & \ddots & & & \vdots \\
0 & \cdots & 0 & \left(\widetilde{\Delta}^{C}\right)^{2} & 0 & \left(\widetilde{\Delta}^{P}\right)^{2} \\
0 & \cdots & 0 & 0 & \left(\widetilde{\Delta}^{C}\right)^{2} & 0 \\
0 & \cdots & 0 & 0 & 0 & \left(\widetilde{\Delta}^{C}\right)^{2}
\end{array}\right)=\sum_{k=1}^{c} f_{k} \tilde{s}_{k} .
$$


Notice that $\left(\widetilde{\Delta}^{C}\right)^{2}=\sum_{k=1}^{c} f_{k} \tilde{t}_{k} \otimes P$ and $\left(\widetilde{\Delta}^{P}\right)^{2}=\sum_{k=1}^{c} f_{k} C \otimes \tilde{r}_{k}$. We can now see that

$$
s_{k}=\left(\begin{array}{cccccc}
t_{k} \otimes P & 0 & C \otimes r_{k} & 0 & \cdots & 0 \\
0 & t_{k} \otimes P & 0 & C \otimes r_{k} & \cdots & 0 \\
\vdots & & \ddots & & & \vdots \\
0 & \ldots & 0 & t_{k} \otimes P & 0 & C \otimes r_{k} \\
0 & \ldots & 0 & 0 & t_{k} \otimes P & 0 \\
0 & \ldots & 0 & 0 & 0 & t_{k} \otimes P
\end{array}\right)
$$

and thus the complex $P$ provides the nilpotent part of $s_{k}$.

\subsection{Examples}

First we give an example that demonstrates Theorem 3.5, i.e. that if $p(t)$ is a polynomial of Eisenbud operators of a totally acyclic complex $C$, then the support variety of Cone $(p(t))$ is $V(Q, f, C, C) \cap Z(p(t))$.

Example 4.3.1. Let $R=\mathbb{C}[[x, y, z]] /\left(x^{2}, y^{2}, z^{2}\right)$ and let $C$ be the complex

$$
\begin{gathered}
C: \cdots \stackrel{d_{n+1}^{C}}{\longrightarrow} R^{3} \stackrel{d_{n}^{C}}{\longrightarrow} R^{3} \stackrel{d_{n-1}^{C}}{\longrightarrow} R^{3} \stackrel{d_{n-2}^{C}}{\longrightarrow} \cdots \\
\text { where } d_{n}^{C}=\left(\begin{array}{ccc}
x & 0 & 0 \\
0 & y & 0 \\
0 & 0 & z
\end{array}\right) \text { for all } n .
\end{gathered}
$$

Then

$$
\left(\tilde{d}^{C}\right)^{2}=\left(\begin{array}{ccc}
x^{2} & 0 & 0 \\
0 & y^{2} & 0 \\
0 & 0 & z^{2}
\end{array}\right)=x^{2} \widetilde{t_{1}}+y^{2} \widetilde{t_{2}}+z^{2} \widetilde{t_{3}}
$$


and so we have

$$
t_{1}=\left(\begin{array}{lll}
1 & 0 & 0 \\
0 & 0 & 0 \\
0 & 0 & 0
\end{array}\right), t_{2}=\left(\begin{array}{lll}
0 & 0 & 0 \\
0 & 1 & 0 \\
0 & 0 & 0
\end{array}\right), t_{3}=\left(\begin{array}{lll}
0 & 0 & 0 \\
0 & 0 & 0 \\
0 & 0 & 1
\end{array}\right) .
$$

Therefore

$$
\operatorname{Ann}\left(\bigoplus_{i \geq 0} \operatorname{Hom}_{R}\left(C, \Sigma^{i} C\right) \otimes_{R} \mathbb{k}\right)=\left(\chi_{1} \chi_{2}, \chi_{2} \chi_{3}, \chi_{1} \chi_{3}\right),
$$

thus the support variety of $C$ is

$$
V(Q, f, C, C)=Z\left(\chi_{1} \chi_{2} \chi_{3}\right)
$$

i.e. the union of the $x, y$, and $z$ axes.

Remark 4.3.2. Notice that $C=D \oplus E \oplus F$ where

$$
\begin{aligned}
& D: \cdots \stackrel{x}{\longrightarrow} R \stackrel{x}{\longrightarrow} R \stackrel{x}{\longrightarrow} R \stackrel{x}{\longrightarrow} \cdots \\
& E: \cdots \stackrel{y}{\longrightarrow} R \stackrel{y}{\longrightarrow} R \stackrel{y}{\longrightarrow} R \stackrel{y}{\longrightarrow} \cdots \\
& F: \cdots \stackrel{z}{\longrightarrow} R \stackrel{z}{\longrightarrow} R \stackrel{z}{\longrightarrow} R \stackrel{z}{\longrightarrow} \cdots
\end{aligned}
$$

and so the support varieties of $D, E$, and $F$ are easily computed to be

$$
\begin{aligned}
& V(Q, f, D, D)=Z\left(\chi_{2}, \chi_{3}\right), \\
& V(Q, f, E, E)=Z\left(\chi_{1}, \chi_{3}\right), \\
& V(Q, f, F, F)=Z\left(\chi_{1}, \chi_{2}\right),
\end{aligned}
$$

i.e. the $x, y$, and $z$ axes respectively. Thus we have

$$
V(Q, f, C, C)=V(Q, f, D, D) \bigcup V(Q, f, E, E) \bigcup V(Q, f, F, F)
$$


Now consider the complex Cone $(p)$ where $p=t_{1}-t_{2}-2 t_{3}$. This complex has differentials given by

$$
d^{\operatorname{Cone}\left(t_{1}\right)}=\left(\begin{array}{cccccc}
x & 0 & 0 & 0 & 0 & 0 \\
0 & y & 0 & 0 & 0 & 0 \\
0 & 0 & z & 0 & 0 & 0 \\
1 & 0 & 0 & -x & 0 & 0 \\
0 & -1 & 0 & 0 & -y & 0 \\
0 & 0 & -2 & 0 & 0 & -z
\end{array}\right) .
$$

Squaring this we have

$$
\left(d^{\operatorname{Cone}(p)}\right)^{2}=\left(\begin{array}{cccccc}
x^{2} & 0 & 0 & 0 & 0 & 0 \\
0 & y^{2} & 0 & 0 & 0 & 0 \\
0 & 0 & z^{2} & 0 & 0 & 0 \\
0 & 0 & 0 & x^{2} & 0 & 0 \\
0 & 0 & 0 & 0 & y^{2} & 0 \\
0 & 0 & 0 & 0 & 0 & z^{2}
\end{array}\right) .
$$

Therefore the Eisenbud operators of the cone are given by matrices of the form $s_{n}=\left(\begin{array}{cc}t_{n} & 0 \\ 0 & t_{n}\end{array}\right)$. Thus with the homotopy maps $\lambda=\left(\begin{array}{llllll}0 & 0 & 0 & 1 & 0 & 0 \\ 0 & 0 & 0 & 0 & 1 & 0 \\ 0 & 0 & 0 & 0 & 0 & 1 \\ 0 & 0 & 0 & 0 & 0 & 0 \\ 0 & 0 & 0 & 0 & 0 & 0 \\ 0 & 0 & 0 & 0 & 0 & 0\end{array}\right)$, we have $\lambda \circ d^{\operatorname{Cone}(p)}-d^{\operatorname{Cone}(p)} \circ \lambda=s_{1}-s_{2}-2 s_{3}$. Therefore the support variety of Cone $(p)$ is 


$$
\begin{gathered}
V\left(Q, f, \operatorname{Cone}(p), \text { Cone }(p)=Z\left(\operatorname{Ann} \bigoplus_{i \geq 0} \operatorname{Hom}\left(\operatorname{Cone}(p), \Sigma^{i} \operatorname{Cone}(p)\right)\right)\right. \\
=Z\left(\chi_{1} \chi_{2} \chi_{3}, \chi_{1}-\chi_{2}-2 \chi_{3}\right)=\{0\} .
\end{gathered}
$$

We now give an example of a complex where one of the nonzero Eisenbud operators squares to zero.

Example 4.3.3. Let $R=\mathbb{k}\left[\left[x_{1}, \ldots, x_{5}\right]\right] /\left(x_{1} x_{2}, x_{3} x_{4}\right)$ where $\mathbb{k}$ is an algebraically closed field. Consider the complex $C \in \mathrm{K}_{\mathrm{tac}}(R)$ given by

$$
C: \cdots \stackrel{d_{n+1}^{C}}{\longrightarrow} R^{8} \stackrel{d_{n}^{C}}{\longrightarrow} R^{8} \stackrel{d_{n-1}^{C}}{\longrightarrow} R^{8} \stackrel{d_{n-2}^{C}}{\longrightarrow} \cdots
$$

where the differentials are given by

$$
d_{n}^{C}=\left(\begin{array}{cccccccc}
-x_{1} & 0 & 0 & -x_{5} & 0 & 0 & 0 & x_{4} \\
-x_{2} & 0 & -x_{5} & 0 & 0 & 0 & x_{4} & 0 \\
0 & 0 & x_{1} & -x_{2} & 0 & x_{4} & 0 & 0 \\
0 & 0 & 0 & x_{2} & x_{4} & 0 & 0 & 0 \\
0 & -x_{1} & 0 & x_{3} & 0 & 0 & 0 & 0 \\
0 & -x_{2} & x_{3} & 0 & 0 & 0 & 0 & 0 \\
0 & x_{4} & 0 & 0 & 0 & 0 & 0 & 0 \\
x_{3} & x_{5} & 0 & 0 & 0 & 0 & 0 & 0
\end{array}\right)
$$


if $n$ is even and

$$
d_{n}^{C}=\left(\begin{array}{cccccccc}
0 & 0 & 0 & 0 & 0 & 0 & -x_{5} & x_{4} \\
0 & 0 & 0 & 0 & 0 & 0 & x_{3} & 0 \\
0 & 0 & 0 & 0 & 0 & x_{4} & x_{2} & 0 \\
0 & 0 & 0 & 0 & x_{4} & 0 & x_{1} & 0 \\
0 & 0 & 0 & x_{3} & -x_{2} & 0 & 0 & 0 \\
0 & 0 & x_{3} & 0 & x_{2} & -x_{1} & 0 & 0 \\
0 & x_{3} & 0 & 0 & 0 & x_{5} & 0 & x_{2} \\
x_{3} & 0 & 0 & 0 & x_{5} & 0 & 0 & x_{1}
\end{array}\right)
$$

if $n$ is odd.

Then

$$
t_{1}=\left(\begin{array}{llllllll}
0 & 0 & 0 & 0 & 0 & 0 & 0 & 0 \\
0 & 0 & 0 & 0 & 0 & 0 & 0 & 0 \\
0 & 0 & 0 & 0 & 0 & 0 & 0 & 0 \\
0 & 0 & 0 & 0 & 0 & 0 & 1 & 0 \\
0 & 0 & 0 & 0 & 0 & 0 & 0 & 0 \\
0 & 0 & 0 & 0 & 0 & 0 & 0 & 0 \\
0 & 0 & 0 & 0 & 0 & 0 & 0 & 0 \\
0 & 0 & 0 & 0 & 0 & 0 & 0 & 0
\end{array}\right)
$$


in even degrees and

$$
t_{1}=\left(\begin{array}{llllllll}
0 & 0 & 0 & 0 & 0 & 0 & 0 & 0 \\
0 & 0 & 0 & 0 & 0 & 0 & 0 & 0 \\
0 & 0 & 0 & 0 & 0 & 0 & 0 & 0 \\
0 & 0 & 0 & 0 & 0 & 0 & 0 & 0 \\
0 & 1 & 0 & 0 & 0 & 0 & 0 & 0 \\
0 & 0 & 0 & 0 & 0 & 0 & 0 & 0 \\
0 & 0 & 0 & 0 & 0 & 0 & 0 & 0 \\
0 & 0 & 0 & 0 & 0 & 0 & 0 & 0
\end{array}\right)
$$

in odd degrees. Furthermore we see that $t_{2}=1_{R^{8}}$. Therefore the support variety of $C$ is given by

$$
V(Q, f, C, C)=Z\left(\operatorname{Ann} \bigoplus_{i \geq 0} \operatorname{Hom}\left(C, \Sigma^{i} C\right)\right)=Z\left(t_{1}^{2}\right)=Z\left(t_{1}\right) .
$$

The next example illustrates the process discussed in the previous section to create nilpotent Eisenbud operators.

Example 4.3.4. Let $R=k[[x, y]] /\left(x^{2}, y^{2}\right)$ and consider the totally acyclic $R$-complex

$$
C: \cdots \stackrel{x}{\longrightarrow} R \stackrel{x}{\longrightarrow} R \stackrel{x}{\longrightarrow} R \stackrel{x}{\longrightarrow} \cdots
$$

and the perfect complex

$$
P: 0 \longrightarrow R \stackrel{y}{\longrightarrow} R \stackrel{y}{\longrightarrow} R \stackrel{y}{\longrightarrow} R \longrightarrow 0 \text {. }
$$

Then we have the totally acyclic complex

$$
C \otimes_{R} P: \cdots \stackrel{d_{n}^{C \otimes_{R} P}}{\longrightarrow} R^{4} \stackrel{d_{n}^{C \otimes_{R} P}}{\longrightarrow} R^{4} \stackrel{d_{n}^{C \otimes_{R} P}}{\longrightarrow} R^{4} \stackrel{d_{n}^{C \otimes_{R} P}}{\longrightarrow} \cdots
$$


with differentials

$$
d_{n}^{C \otimes_{R} P}=\left(\begin{array}{cccc}
x & (-1)^{n+1} y & 0 & 0 \\
0 & x & (-1)^{n} y & 0 \\
0 & 0 & x & (-1)^{n+1} y \\
0 & 0 & 0 & x
\end{array}\right) .
$$

Lifting the differential to $Q$ and squaring it yields

$$
\left(d^{C \otimes_{R} P}\right)^{2}=\left(\begin{array}{cccc}
x^{2} & 0 & y^{2} & 0 \\
0 & x^{2} & 0 & y^{2} \\
0 & 0 & x^{2} & 0 \\
0 & 0 & 0 & x^{2}
\end{array}\right)=\sum_{k=1} f_{k} \widetilde{s_{k}}
$$

and so the Eisenbud operators are $s_{1}=\left(\begin{array}{cccc}1 & 0 & 0 & 0 \\ 0 & 1 & 0 & 0 \\ 0 & 0 & 1 & 0 \\ 0 & 0 & 0 & 1\end{array}\right)$ and $s_{2}=\left(\begin{array}{llll}0 & 0 & 1 & 0 \\ 0 & 0 & 0 & 1 \\ 0 & 0 & 0 & 0 \\ 0 & 0 & 0 & 0\end{array}\right)$. Notice that $s_{1}^{r} \neq 0$ for any $r \in \mathbb{N}$ while $s_{1}^{3}=0$ and $s_{1}^{2} \neq 0$. Thus the support variety is $V\left(Q, f, C \otimes_{R} P, C \otimes_{R} P\right)=Z\left(\chi_{2}\right)$.

While we have examples of totally acyclic complexes with nilpotent Eisenbud operators, none of these complexes are themselves mapping cones of Eisenbud operators. In order to create a mapping cone Cone $(p(t))$ with nilpotent Eisenbud operators, we need the bottom left corner of the lifted and squared differential to be nonzero. This will only occur if the polynomial $p(t)$ has entries in the maximal ideal. Thus we would like to find an example of a totally acyclic complex with an Eisenbud operator with elements contained in the maximal ideal. The following example looks promising at first, but the Eisenbud operator in question turns out to be homotopic to zero. 
Example 4.3.5. Let $R=\mathbb{k}[[x, y]] /\left(x^{a}-y^{b}, x y\right)$ and let $C$ be the period-two complex

$$
C: \cdots \stackrel{d_{n}^{C}}{\longrightarrow} R \stackrel{d_{n-1}^{C}}{\longrightarrow} R \stackrel{d_{n-2}^{C}}{\longrightarrow \cdots}
$$

where $d_{n}^{C}=x+y$ if $n$ is even and $d_{n}^{C}=x^{a-1}-y^{b-1}$ if $n$ is odd. Then

$$
\left(\tilde{d}^{C}\right)^{2}=x^{a}-y^{b}+y x^{a-1}-x y^{b-1}=\left(x^{a}-y^{b}\right)(1)+(x y)\left(x^{a-2}-y^{b-2}\right) .
$$

Thus $t_{1}=1$ and $t_{2}=x^{a-2}-y^{b-2}$.

Now consider homotopy maps $\lambda_{n}=0$ if $n$ is even and $\lambda_{n}=x^{a-3}-y^{b-3}$ if $n$ is odd. Since $x y=0$, we have

$$
t_{2}=x^{a-2}-y^{b-2}=\left(x^{a-3}-y^{b-3}\right)(x+y)=d_{n+1}^{\Sigma^{2} C} \lambda_{n}+\lambda_{n-1} d_{n}^{C} .
$$

Therefore $t_{2}$ is homotopic to zero and hence we can now see that the support variety of $C$ is given by

$$
V(Q, f, C, C)=Z\left(\chi_{2}\right)
$$




\section{Conclusions and Future Work}

We chose to study support and rank varieties in $\mathrm{K}_{\text {tac }}(R)$ in order use the triangulated structure of this category to give elegant proofs of a few classic results. In particular, we spent most of our time studying the differentials of mapping cones of Eisenbud operators. Understanding these cones allowed us to give very direct proofs about the finiteness of modules of cohomology operators. This finite generation result allowed us to show that the Avrunin-Scott theorem holds in $\mathrm{K}_{\mathrm{tac}}(R)$. We were also able to study the Eisenbud operators of the cone itself to create a sequence of totally acyclic complexes with the final complex having a desired support variety.

Our next goal is to use the explicit descriptions of mapping cones in this category to study the scheme structure of support varieties. Our proof of the realizability of support varieties requires Hilbert's Nullstellensatz. Thus we only have a description of $\operatorname{Ann} \operatorname{Hom}_{R}\left(\operatorname{Cone}(p), \Sigma^{i} \operatorname{Cone}(p)\right)$ up to radical. We know that if $q \in \operatorname{Ann} \operatorname{Hom}_{R}\left(C, \Sigma^{i} C\right)$, then $q^{2} \in \operatorname{Ann} \operatorname{Hom}_{R}\left(\operatorname{Cone}(p), \Sigma^{i} \operatorname{Cone}(p)\right)$. We would like to know if this can be strengthened so that $q \in \operatorname{Ann} \operatorname{Hom}_{R}\left(\operatorname{Cone}(p), \Sigma^{i} \operatorname{Cone}(p)\right)$ or if instead we can find an example of a mapping cone with a nipotent polynomial of Eisenbud operators.

In order to find a mapping cone with a nilpotent polynomial of Eisenbud operators, the bottom left corner of the lifted and squared differential cannot be zero. We already know that for a polynomial $p(t)$ of Eisenbud operators, the mapping cone Cone $(p(t))$ has Eisenbud operators $s_{i}$ such that $p(s) \sim 0$. This is precisely due to the fact that the bottom left corner of $p(s)$ is always zero. However, we do not know if this is the case for a generic polynomial $q(s)$ of Eisenbud operators of Cone $(p(t))$. 
The bottom left corner of the lifted squared differential is roughly of the form $\tilde{d}^{C} \tilde{p}(t)-\tilde{p}(t) \tilde{d}^{C}$. This term is zero when tensored down to $R$ and thus is equal to a linear combination of the $f_{i}$ 's which are in the maximal ideal. Thus if $\tilde{d}^{C} \tilde{p}(t)-\tilde{p}(t) \tilde{d}^{C}$ is not zero, $p(t)$ must have entries in the maximal ideal. Therefore the only hope of finding a mapping cone with a nilpotent polynomial of Eisenbud operators is to first find a totally acyclic complex with an Eisenbud operator that has entries in the maximal ideal. So far the only example we have of such an Eisenbud operator turned out to be homotopic to zero. Therefore we would like to pose the following question: does there exist a totally acyclic complex with an Eisenbud operator $t_{i}$ such that for any $s_{i}$ that is homotopic to $t_{i}, s_{i}$ has entries in the maximal ideal?

The category of totally acyclic chain complexes is important to study because of its connection to several other triangulated categories. In [12], Buchweitz showed that for any Gorenstein ring $R, \mathrm{~K}_{\mathrm{tac}}(R)$ is equivalent to the stable category of maximal Cohen-Macaulay modules over $R$, the singularity category, i.e. the derived category modulo perfect complexes. The derived category of coherent sheaves plays an important role in homological mirror symmetry and thus has applications in string theory. Furthermore, the category of matrix factorizations is also equivalent to $\mathrm{K}_{\mathrm{tac}}(R)$ if $R$ is a hypersurface and it has known applications in Landau-Ginzberg models. We hope to use our theory of support and rank varieties in $\mathrm{K}_{\mathrm{tac}}(R)$ to provide new insights in these areas. 


\section{References}

[1] L.L. Avramov, Modules of finite virtual projective dimension, Invent. Math. 96 (1989), no. 1, 71-101.

[2] L.L Avramov and R.O. Buchweitz, Support varieties and cohomology over complete intersections, Invent. Math. 142 (2000), no. 2, 285-318.

[3] L.L. Avramov, V. Gasarov, and I. Peeva, Complete intersection dimension, Publ. Math. I.H.E.S, 86 (1997), 67-114.

[4] L.L. Avramov and S. Iyengar, Constructing modules with prescribed cohomological support, Illinois. J. Math. 51, 2007, Special Issue dedicated to Phillip Griffith, to appear.

[5] L.L. Avramov and A. Martsinkovsky, Absolute, relative, and Tate cohomology of modules of finite Gorenstein dimension, Proc. London Math. Soc. (3) 85 (2002), no. $2,393-440$.

[6] G.S. Avrunin and L.L. Scott, Quillen stratification for modules, Invent. Math., 66 (1982) 211-286.

[7] D. Benson, S. Iyengar, and H. Krause, Local Cohomology and Support for Triangulated Categories, Ann. Sci. Ecole Norm. Sup, 41 (2008) 575-621.

[8] P. Bergh, On support varieties for modules over complete intersections, Proc. Amer. Math. Soc. 135, no. 12, 2007, pg.3795-3803.

[9] P. Bergh and D. Jorgensen, Support Varieties Over Complete Intersections Made Easy, arXiv:1509.07828 [math.AC] (2015).

[10] P. Bergh, D. Jorgensen, and F. Moore, Totally acyclic approximations, arXiv:1606.07976 [math.ac] (2016). 
[11] W. Bruns and J. Herzog, Cohen-Macaulay rings: Revised Edition, Cambridge University Press, New York NY, 1998.

[12] R.O. Buchweitz, Maximal Cohen-Macaulay Modules and Tate-Cohomology Over Gorenstein Rings, University of Hanover, (1986).

[13] J.F. Carlson, The varieties and the cohomology ring of a module, J. Algebra 85 (1983), no. 1, 104-143.

[14] E.C. Dade, Endo-permutation modules over p-groups. II. Ann. of Math. (2) 108 (1978), no. 2, 317-346.

[15] D. Eisenbud, Homological algebra on a complete intersection, with an application to group representations, Trans. Amer. Math. Soc. 260 (1980), no. 1, 35-64.

[16] T. Fritz, Notes on Triangulated Categories, arXiv:1407.3765 [math.KT] (2014).

[17] T.H. Gulliksen, A change of ring theorem with applications to Poincaré series and intersection multiplicity, Math. Scand. 34 (1974), 167-183.

[18] A. Neeman, Triangulated Categories, Annals of Mathematics Studies: Princeton University Press, Princeton NJ, (2001).

[19] D. Quillen, The Spectrum of an equivariant cohomology ring: I,II, Ann. of Math. (2) 94 (1971), 549-602.

[20] J.J. Rotman, An Introduction to Homological Algebra: Second Edition, Springer Science+Business Media, LLC., New York NY, (2009).

[21] J.L. Verdier Des cat'egories d'eriv'ees des cat'egories ab'eliennes, later published as Ast'erisque, SMF 239 (1996).

[22] C.A. Weibel An Introduction to homological algebra, Cambridge University Press, Cambridge UK, (1997). 


\section{Biographical Statement}

Nathan Steele was born on March 29th, 1989 in Bethesda Maryland to Ricky and Diana Steele and is the second of two sons. He graduated from Luther High School in Luther Oklahoma in 2007. He then attended the University of Central Oklahoma in Edmond Oklahoma and graduated with a Bachelor of Science degree in mathematics in May of 2011. After completion of his Bachelor's degree, Nathan moved to Arlington Texas and began doctoral studies at the University of Texas at Arlington. In August 2016, he completed his dissertation in mathematics under the direction of Dr. David Jorgensen.

Nathan's research interests include commutative algebra, homological algebra, and algebraic geometry. He also maintains a strong interest in the pedagogy and philosophy of mathematics. 